\title{
Positron Emission Tomography Based Elucidation of the Enhanced Permeability and Retention Effect in Dogs with Cancer Using Copper-64 Liposomes
}

Hansen, Anders Elias; Petersen, Anncatrine Luisa; Henriksen, Jonas Rosager; Børresen, Betina;

Rasmussen, Palle; Elema, Dennis Ringkjøbing; Rosenschöld, Per Munck af; Kristensen, Annemarie T.;

Kjær, Andreas; Andresen, Thomas Lars

\section{Published in: \\ A C S Nano}

Link to article, DOI:

10.1021/acsnano.5b01324

Publication date:

2015

Document Version

Peer reviewed version

Link back to DTU Orbit

Citation (APA):

Hansen, A. E., Petersen, A. L., Henriksen, J. R., Børresen, B., Rasmussen, P., Elema, D. R., Rosenschöld, P. M. A., Kristensen, A. T., Kjær, A., \& Andresen, T. L. (2015). Positron Emission Tomography Based Elucidation of the Enhanced Permeability and Retention Effect in Dogs with Cancer Using Copper-64 Liposomes. A C S Nano, 9(7), 6985-6995. https://doi.org/10.1021/acsnano.5b01324

\section{General rights}

Copyright and moral rights for the publications made accessible in the public portal are retained by the authors and/or other copyright owners and it is a condition of accessing publications that users recognise and abide by the legal requirements associated with these rights.

- Users may download and print one copy of any publication from the public portal for the purpose of private study or research.

- You may not further distribute the material or use it for any profit-making activity or commercial gain

- You may freely distribute the URL identifying the publication in the public portal 


\section{Positron Emission Tomography based Elucidation of the Enhanced Permeability and Retention Effect in Dogs with Cancer using Copper-64 Liposomes}

Anders E. Hansen ${ }^{1,2}$, Anncatrine L. Petersen ${ }^{1}$, Jonas R. Henriksen ${ }^{1,3}$, Betina Boerresen, ${ }^{4}$ Palle Rasmussen $^{1,5}$, Dennis R. Elema ${ }^{1,5}$, Per Munch af Rosenschöld ${ }^{6}$, Annemarie T. Kristensen ${ }^{4}$, Andreas Kjor $^{2}$, Thomas L. Andresen ${ }^{1}$.

${ }^{1}$ Center for Nanomedicine and Theranostics, DTU Nanotech, Technical University of Denmark, Building 423, DK-2800 Lyngby, Denmark; ${ }^{2}$ Department of Clinical Physiology, Nuclear Medicine \& PET, and Cluster for Molecular Imaging, Rigshospitalet, Copenhagen University Hospital and Faculty of Health Sciences, University of Copenhagen, Blegdamsvej 3B, DK-2200 Copenhagen, Denmark; ${ }^{3}$ DTU Chemistry, Technical University of Denmark, Building 206, DK-2800 Lyngby, Denmark; ${ }^{4}$ Department of Veterinary Clinical and Animal Sciences, Faculty of Health and Medical Sciences, University of Copenhagen, Dyrlaegevej 16, DK-1870 Frederiksberg, Denmark; ${ }^{5}$ DTU Nutech, Hevesy Laboratory, Technical University of Denmark, Frederiksborgvej 399, DK-4000 Roskilde, Denmark; ${ }^{6}$ Radiation Medicine Research Center, Department of Radiation Oncology, Rigshospitalet, Copenhagen University Hospital, Blegdamsvej 9, DK-2100 Copenhagen, Denmark;

E-mail: thomas.andresen@nanotech.dtu.dk

RECEIVED DATE (to be automatically inserted after your manuscript is accepted if required according to the journal that you are submitting your paper to)

*Corresponding author contact details, Phone: +45 45258168, Fax: 4588 7762, E-mail: Thomas.andresen@nanotech.dtu.dk 
ABSTRACT. Since the first report of the enhanced permeability and retention (EPR) effect, the research in nanocarrier based anti-tumor drugs has been intense. The field has been devoted to treatment of cancer by exploiting EPR-based accumulation of nanocarriers in solid tumors, which for many years was considered to be a ubiquitous phenomenon. However, the understanding of differences in the EPR-effect between tumor types, heterogeneities within each patient group, and dependency on tumor development stage in humans is sparse. It is therefore important to enhance our understanding of the EPR-effect in large animals and humans with spontaneously developed cancer. In the present paper, we describe a novel loading method of copper-64 into PEGylated liposomes and use these liposomes to evaluate the EPR-effect in 11 canine cancer patients with spontaneous solid tumors by PET/CT imaging. We thereby provide the first high-resolution analysis of EPR-based tumor accumulation in large animals. We find that the EPR-effect is strong in some tumor types but cannot be considered a general feature of solid malignant tumors since we observed a high degree of accumulation heterogeneity between tumors. Six of seven included carcinomas displayed high uptake levels of liposomes, whereas one of four sarcomas displayed signs of liposome retention. We conclude that nanocarrier-radiotracers could be important in identifying cancer patients that will benefit from nanocarrier-based therapeutics in clinical practice.

KEYWORDS. Nanomedicine, cancer, EPR-effect, drug delivery, imaging, nanoparticles, liposomes

The therapeutic potential of nanocarrier based chemotherapeutics for the treatment of cancer is considered huge and considerable success has been achieved since its introduction. ${ }^{1,2}$ Many of the developed nanocarrier systems are considered to accumulate in tumors based on the enhanced permeability and retention (EPR)-effect, ${ }^{3}$ which in nanomedicine research has been considered a universal feature of solid malignant tumors that can serve as the basis for passive tumor targeting of therapeutic and diagnostic nanoparticles. ${ }^{4-6}$

Solid tumors growing beyond a size of a few millimeters depend on successful formation of neoangiogenic blood vessels to meet their oxygen and energy requirements, and for removal of waste products. $^{7,8}$ The blood vessels of malignant tumors display several anatomical and pathophysiological differences relative to the vessels in normal tissues. Tumors display a poorly organized vascular architecture, which have been reported to include wide endothelial fenestrations and absent lymphatic drainage. ${ }^{5,9,10}$ These features form the basis for the EPR-based extravasation of 
nano-sized particles into tumor tissue. ${ }^{5,9}$ The heterogeneous tumor microenvironment contains wellcharacterized loco-regional features that may affect the accumulation of nanoparticles in tumors, including poorly perfused regions of hypoxia ${ }^{11}$ and regional activity of vasoactive proteins. ${ }^{12,13}$

Massive efforts are currently directed towards the development of nanocarriers that can deliver drugs to cellular and subcellular targets in tumors. It has been argued that the therapeutic benefit of marketed nanotherapeutics for cancer relates to reduced toxicity, e.g. reduced cardiovascular toxicity of Doxil relative to doxorubicin rather than an improved therapeutic effect. ${ }^{14}$ To provide new evidence of the therapeutic potential of nanocarrier systems for treating cancer, an important question to address is related to the EPR-effect. Current knowledge on the ability of nanocarriers to deliver chemotherapeutics to solid tumors mainly originates from quantitative estimation of tumor targeting in experimental models, primarily xenografts in mice, and the actual data in cancer patients is scarce. It is remarkable that despite the theoretical potential and obtained results, only limited efforts have been made to investigate tumor targeting of nanoparticle formulations in spontaneous tumors in large animals. Current knowledge on the EPR-effect in human tumors is based on studies of low-resolution single photon imaging techniques of radiolabeled liposomes. ${ }^{15-18}$ Liposome uptake has been investigated in a Kaposi sarcoma and a lymphoma patient. ${ }^{16}$ Furthermore, targeting and delineation has been investigated in eight high-grade gliomas ${ }^{17}$ and positive tumor identification using radiolabeled phospholipid vesicles in 22 of 24 patients suffering from a few malignancies. ${ }^{18}$ Gamma camera and single-photon emission computed tomography (SPECT) imaging of ${ }^{111}$ In-DTPA-labeled pegylated liposomes was also performed in 17 patients suffering from head and neck, breast, bronchus and cervix cancer and two glioma patients, with 12 tumors being identified by gamma camera imaging and 15 tumors identified by SPECT. ${ }^{15}$ However, all these studies only provide the conclusion that tumors were visible due to presence of radiolabeled liposomes in the tumor and did not report direct quantitative evidence of the EPReffect, as we will show and discuss in the present study. Thus, there is a strong need for studies in large animals with spontaneous syngeneic tumors, with a microenvironment and vasculature that is comparable to human tumors, as this will elucidate the tumor targeting potential of nanocarriers and thereby guide future development of nanoparticle-based drug delivery systems.

In this study, we report a new and highly efficient method for labeling PEGylated liposomes with copper-64 and utilize positron emission tomography/computed tomography (PET/CT) imaging to evaluate liposome tumor accumulation including heterogeneity and pharmacokinetics in family owned dogs with spontaneous solid tumors. The new loading method is based on an un-assisted 
transport of copper-64 into liposomes, which is surprisingly efficient as diffusion of divalent cations such as $\mathrm{Cu}^{2+}$ across lipid bilayers is highly un-expected. We utilize the developed copper-64 liposome imaging agents for studying the EPR-effect in dogs with cancer as the biological features of cancer in dogs is similar to humans and their size and heterogeneous microenvironment make them attractive models for comparative research. ${ }^{19}$ PEGylated liposomes with the same lipid composition as the clinically used Doxil formulation, radiolabeled with copper-64 ([Cu-64]liposomes), were utilized in the study. PET/CT imaging allows for the acquisition of dynamic imaging data and determination of direct quantitative uptake data within specific regions of interest (ROI). ${ }^{20,21}$ The high detection sensitivity and spatial resolution of PET compared to SPECT and sensitivity compared to magnetic resonance (MR) imaging makes PET superior for quantitative studies of nanoparticle biodistribution..$^{20,22,23}$ In addition, PET is a true quantitative technique.

\section{Results}

Preparation of liposome imaging probes by un-assisted loading of Copper-64. In the present work, DSPC:CHOL:DSPE-PEG ${ }_{2000}(56.5: 38.2: 5.3)$ liposomes were prepared with the high affinity copper chelator DOTA encapsulated. These liposomes were used for loading of $[\mathrm{Cu}-64]^{2+}$ by a new un-assisted loading method without the use of ionophores or ion transporters, ${ }^{24}$ as schematically illustrated in Figure 1a. In this method, copper-64 chloride is added to liposome solutions and postloading purification is not needed due to very high loading efficiencies, which is important for PET imaging due to the short half-life of PET isotopes (e.g. copper-64 has a $12.7 \mathrm{~h}$ half-life). Unassisted loading of $[\mathrm{Cu}-64]^{2+}$ into the liposomes was possible by increasing the temperature during loading to $55^{\circ} \mathrm{C}$, which was surprising as divalent cations are not generally expected to cross lipid bilayers. ${ }^{24}$ The $[\mathrm{Cu}-64]^{2+}$ was trapped inside the liposome by the encapsulated DOTA. Liposome loading kinetics of $[\mathrm{Cu}-64]^{2+}$ was evaluated using radio-TLC (Figure 1b) and size exclusion chromatography. Full loading was observed at $55{ }^{\circ} \mathrm{C}$ within $60 \mathrm{~min}$ after addition of 64-copper chloride, where $>98 \%$ of the copper was loaded in 10 independent experiments. The $[\mathrm{Cu}-64]^{2+}$ loading was surprisingly fast where $38 \% \pm 3 \%$ was loaded within the first minute after addition. The [Cu-64]-liposomes was used without further purification in the dog study, which was possible due to the very high loading efficiency. 

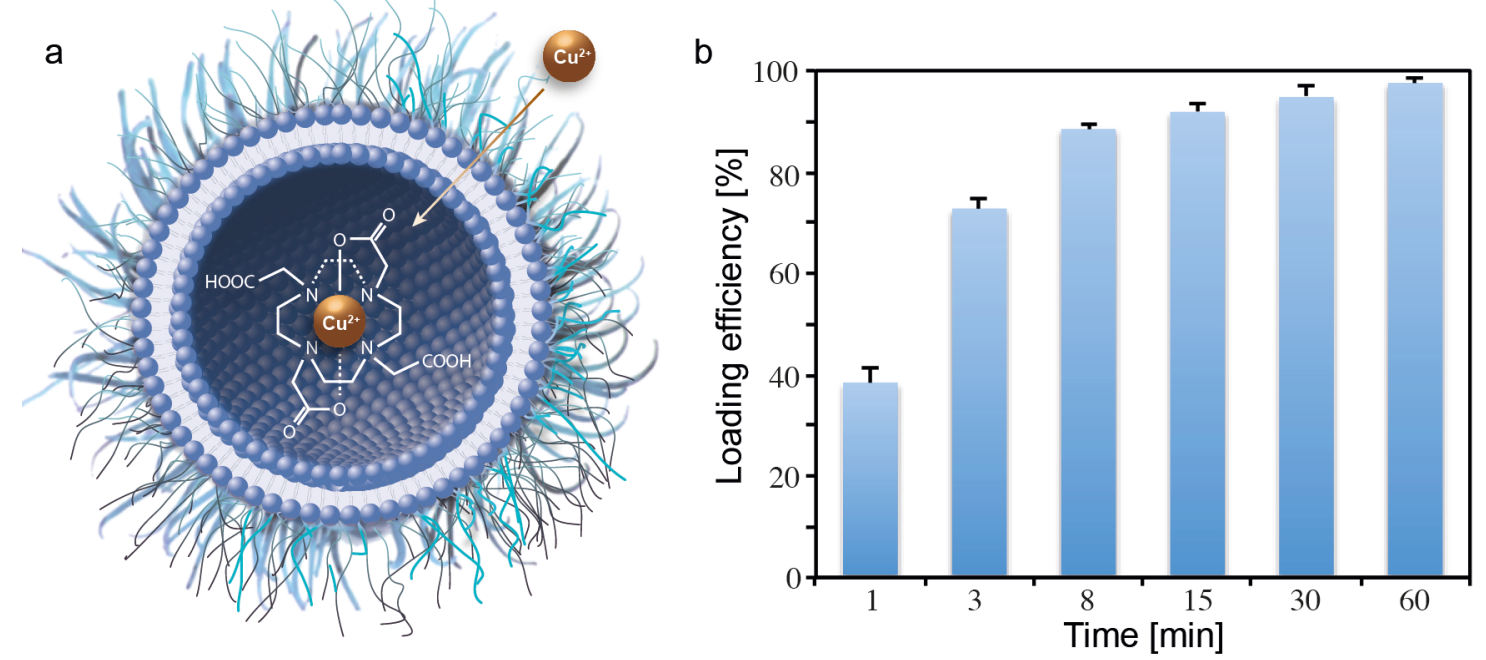

Figure 1. (a) Schematic illustration of Loading of $[\mathrm{Cu}-64]^{2+}$ into liposomes. After addition of $[\mathrm{Cu}-$ $64]^{2+}$ to a liposome solution, $[\mathrm{Cu}-64]^{2+}$ diffuse across the lipid bilayer without the use of ionophores or ion-transporters. [Cu-64] $]^{2+}$ then forms a complex with the encapsulated chelator (DOTA) and is hereby trapped inside the liposome. (b) Loading efficiency of liposomes given as function of time. The loading efficiency, for loading of $[\mathrm{Cu}-64]^{2+}$ into liposomes, was evaluated at $55{ }^{\circ} \mathrm{C}$ by the use of radio-TLC at 1, 3, 8, 15, 30 and $60 \mathrm{~min}$. The liposomes were composed of HSPC:CHOL:DSPE$\mathrm{PEG}_{2000}$ in the molar ratio (56.5:38.2:5.3). The error bars represent SEM $(\mathrm{n}=3)$.

EPR based liposomal accumulation in spontaneous tumors in canine patients. Family owned dogs suffering from spontaneous malignancies were included in the study. All dogs had a histopathological diagnosis based on tumor formalin fixed paraffin embedded HE stained tumor biopsies or sections. [Cu-64]-liposomes with a composition of HSPC/Cholesterol/DSPE-PEG 2000 56.5:38.2:5.3 were used (Doxil lipid composition), which allowed for high-resolution PET imaging of liposome biodistribution. Six of the included $11 \mathrm{dogs}$ were also subjected to $2-\left[{ }^{18} \mathrm{~F}\right]$ fluoro-2deoxy-D-glucose (FDG) PET/CT imaging. FDG PET/CT has a central position for PET imaging of cancer patients and was performed for tumor delineation and staging purposes.

The first [Cu-64]-liposome PET/CT scan was recorded one day after the FDG PET/CT. Dogs had PET/CT scans performed on two consecutive days approximately $24 \mathrm{~h}$ apart, except for $\operatorname{dog} 11$ where the 1-hour [Cu-64]-liposome PET/CT was not performed for technical reasons. The [Cu-64]liposomes were administered by intravenous infusion at increasing rates over a $20 \mathrm{~min}$ period. None of the included dogs displayed any anaphylactic, toxic or adverse reactions. Tumor characteristics and PET uptake of FDG and [Cu-64]-liposomes (standardized uptake value (SUV)) are reported in 
Table 1. [Cu-64]-liposome PET scans were performed dynamically during the initial 0-40 min over the liver and spleen, and hereafter 40-60 min over the tumor (termed 1-hour scan), and again after $24 \mathrm{~h}$ (termed 24-hour scan). The FDG scanned carcinomas displayed higher SUV of FDG relative to the sarcomas, which is in agreement with a previous report on FDG PET in dogs. ${ }^{25}$ All carcinomas displayed increased mean SUV $\left(\mathrm{SUV}_{\text {mean }}\right)$ and particularly maximum SUV $\left(\mathrm{SUV}_{\max }\right)$ of [Cu-64]-liposomes between the 1-hour and 24-hour PET scans, except dog 9 where $\mathrm{SUV}_{\text {mean }}$ did not increase between scans. Oppositely, SUV did not increase for the sarcomas, except for a $\mathrm{SUV}_{\max }$ increase in $\operatorname{dog} 4$.

Table 1. Dog and tumor characteristics, and tumor uptake as standardized uptake values (SUV) for ${ }^{18}$ FDG and [Cu-64]-liposomes

\begin{tabular}{|c|c|c|c|c|c|c|c|c|c|c|c|c|}
\hline Dog & $\begin{array}{l}\text { Age/ } \\
\text { Sex }\end{array}$ & Breed & $\begin{array}{l}\text { BW } \\
(\mathrm{kg})\end{array}$ & $\begin{array}{c}\text { Tumor } \\
\text { type }\end{array}$ & $\begin{array}{c}\text { Tumor } \\
\text { volume } \\
\left(\mathrm{cm}^{3}\right)\end{array}$ & $\begin{array}{c}\text { Tumor } \\
\text { location }\end{array}$ & \multicolumn{2}{|c|}{$\begin{array}{c}{ }^{18} \text { FDG } \\
\text { PET/CT }\end{array}$} & \multicolumn{2}{|c|}{$\begin{array}{c}\text { 1-hour } \\
\text { [64-Cu]-liposome } \\
\text { PET/CT }\end{array}$} & \multicolumn{2}{|c|}{$\begin{array}{c}\text { 24-hour } \\
\text { [64-Cu]-liposome } \\
\text { PET/CT }\end{array}$} \\
\hline 1 & $11 / \mathrm{M}$ & $\begin{array}{l}\text { Golden } \\
\text { Retriever }\end{array}$ & 39 & SCC & 4.0 & Intranasal & 8.7 & 14.5 & 2.1 & 5.5 & 6.1 & 21.3 \\
\hline 2 & $8 / \mathrm{F}$ & $\begin{array}{c}\text { Mixed } \\
\text { breed }\end{array}$ & 28 & AC & 274.5 & $\begin{array}{l}\text { Mammary } \\
\text { glands }\end{array}$ & 4.8 & 17.6 & 0.7 & 3.2 & 1.3 & 11.2 \\
\hline 3 & $5 / \mathrm{F}$ & $\begin{array}{l}\text { Labrador } \\
\text { Retriever }\end{array}$ & 25 & $\mathbf{A C}$ & 19.4 & $\begin{array}{c}\text { Sub- } \\
\text { mandibular }\end{array}$ & 5.3 & 8.4 & 1.3 & 4.2 & 1.6 & 7.7 \\
\hline 4 & $8 / \mathrm{F}$ & Beagle & 12 & STS & 32.6 & $\begin{array}{c}\text { Masticatory } \\
\text { muscle }\end{array}$ & 1.8 & 4.3 & 0.5 & 2.0 & 0.4 & 2.6 \\
\hline 6 & $6 / F$ & $\begin{array}{l}\text { Labrador } \\
\text { Retriever }\end{array}$ & 32 & LS & 4.8 & $\begin{array}{c}\text { Ante- } \\
\text { brachium }\end{array}$ & 2.4 & 3.6 & 0.8 & 2.5 & 0.6 & 1.5 \\
\hline 7 & $12 / \mathrm{M}$ & $\begin{array}{l}\text { Labrador } \\
\text { Retriever }\end{array}$ & 33 & TCC & 22.0 & Intranasal & - & - & 1.4 & 3.7 & 2.6 & 18.7 \\
\hline 8 & $7 / F$ & $\begin{array}{l}\text { Labrador } \\
\text { Retriever }\end{array}$ & 27 & STS & 10.1 & Front paw & - & - & 0.6 & 1.6 & 0.3 & 1.4 \\
\hline 9 & $9 / \mathrm{F}$ & Dachshund & 10 & $\mathbf{A C}$ & 7.1 & $\begin{array}{l}\text { Mammary } \\
\text { glands }\end{array}$ & - & - & 0.7 & 1.7 & 0.7 & 2.0 \\
\hline 10 & $10 / \mathrm{M}$ & Schnauzer & 13 & SCC & 8.0 & Intranasal & - & - & 1.3 & 4.7 & 2.9 & 6.9 \\
\hline 11 & $12 / \mathrm{M}$ & $\begin{array}{l}\text { Golden } \\
\text { Retriever }\end{array}$ & 32 & $\mathrm{AC}$ & 36.6 & Intranasal & - & - & - & - & 1.6 & 10.2 \\
\hline
\end{tabular}

M: Male, F: Female, BW: Bodyweight, SCC: Squamous cell carcinoma, AC: Adenocarcinoma, STS: Soft tissue sarcoma, TCC: Transitional cell carcinoma, LS: Liposarcoma. Carcinomas in bold. Tumor volume determined by PET/CT-scan. 


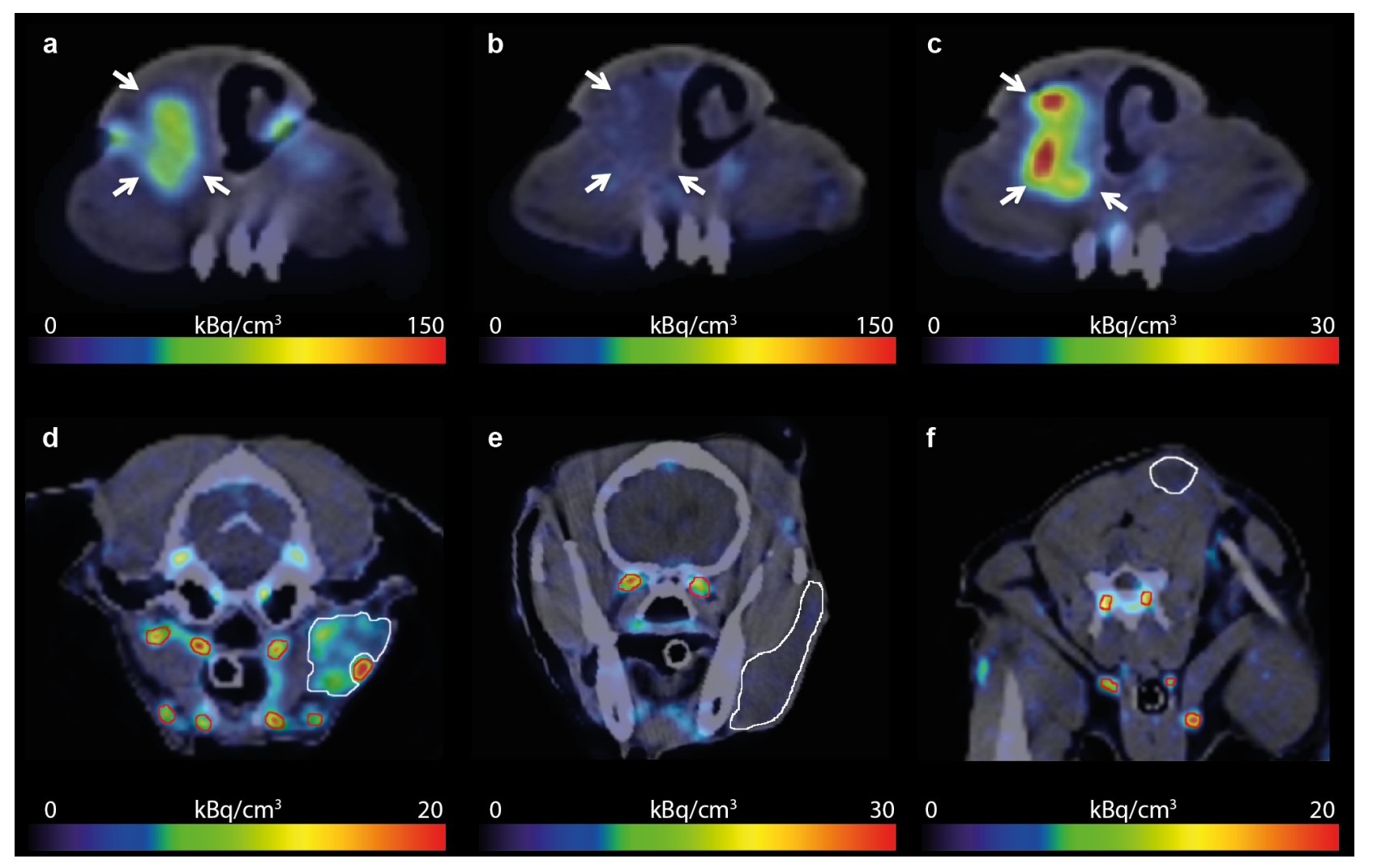

Figure 2. FDG PET/CT and [Cu-64]-liposome PET/CT. PET/CT image of the intranasal squamous cell carcinoma in the right nasal cavity of $\operatorname{dog} 1$ (a-c), illustrating tumor accumulation of FDG (a), [Cu-64]liposome PET/CT $1 \mathrm{~h}$ after liposome infusion (b), [Cu-64]-liposome PET/CT $24 \mathrm{~h}$ after liposome infusion (c). The heterogeneous tumor uptake of the liposomes (24-hour PET/CT) can be visually appreciated on the 24-hour [Cu-64]-liposome PET/CT image of the adenocarcinoma of dog 3 (d). 24-hour [Cu-64]-liposome PET/CT images of the soft tissue sarcomas (STS) in the mandibular region of $\operatorname{dog} 4$ (e) and the neck musculature of $\operatorname{dog} 5$ (f) illustrates the low accumulation in these tumors relative the carcinomas (c,d). Tumors are marked by arrows or delineated by white lines and larger blood vessels by red circles (c-d).

Accumulation levels of [Cu-64]-liposomes were determined by calculating decay corrected percentage of injected dose (\%ID) of [Cu-64]-liposomes in tumors (Table 2). The included carcinomas displayed increased total tumor, mean and maximum \% ID of [Cu-64]-liposomes between the 1-hour and 24-hour PET scans, except dog 9 where a slightly decreased mean and total $\%$ ID/g was observed. The included sarcomas displayed decreased \%ID values, except for a slightly increased maximum \%ID in dog 4 . The tumor mean $\%$ ID $/ \mathrm{kg}$ after a distribution period of only $1 \mathrm{~h}$ is expected to primarily consist of activity in tumor vasculature and ranged from $2.2-11.2$ (mean 4.5, SEM 0.8) \% ID $/ \mathrm{kg}$. 
Table 2. Tumor uptake levels of [Cu-64]-liposomes 1 and 24 hours after injection

\begin{tabular}{|c|c|c|c|c|c|c|}
\hline \multirow[b]{2}{*}{ Dog } & \multicolumn{3}{|c|}{$\begin{array}{c}\text { 1-hour } \\
{[\mathrm{Cu}-64]-\text {-liposome PET/CT }}\end{array}$} & \multicolumn{3}{|c|}{$\begin{array}{c}\text { 24-hour } \\
{[\mathrm{Cu}-64] \text {-liposome PET/CT }}\end{array}$} \\
\hline & $\begin{array}{l}\text { Total } \\
\% \text { ID }\end{array}$ & $\begin{array}{c}\text { Mean } \\
\% \mathrm{ID} / \mathrm{kg}\end{array}$ & $\begin{array}{c}\operatorname{Max} \\
\% \mathrm{ID} / \mathrm{kg}\end{array}$ & $\begin{array}{l}\text { Total } \\
\% \text { ID }\end{array}$ & $\begin{array}{c}\text { Mean } \\
\% \mathrm{ID} / \mathrm{kg}\end{array}$ & $\begin{array}{c}\mathrm{Max} \\
\% \mathrm{ID} / \mathrm{kg}\end{array}$ \\
\hline 1 & 0.02 & 4.6 & 16 & 0.07 & 17 & 59 \\
\hline 2 & 0.61 & 2.5 & 11 & 1.29 & 4.8 & 40 \\
\hline 3 & 0.11 & 5.2 & 17 & 0.13 & 6.5 & 31 \\
\hline 4 & 0.14 & 3.8 & 15 & 0.11 & 3.5 & 21 \\
\hline 5 & 0.03 & 2.5 & 7.7 & 0.02 & 1.5 & 5.7 \\
\hline 6 & 0.01 & 2.3 & 7.9 & 0.01 & 1.8 & 4.7 \\
\hline 7 & 0.10 & 4.2 & 11.2 & 0.18 & 8.1 & 57.1 \\
\hline 8 & 0.03 & 2.2 & 6.1 & 0.01 & 1.1 & 5.3 \\
\hline 9 & 0.09 & 7.4 & 17 & 0.05 & 6.7 & 20.0 \\
\hline 10 & 0.10 & 11.2 & 35.8 & 0.19 & 23.1 & 54.1 \\
\hline 11 & - & - & - & 0.20 & 5.4 & 32.0 \\
\hline
\end{tabular}

Total \%ID: Percentage of injected dose in total. Mean and max \%ID/kg: Mean and maximum percentage of injected dose pr. $\mathrm{kg}$ of tumor tissue determined from liposome PET/CT scans performed $1 \mathrm{~h}$ and $24 \mathrm{~h}$ after injection. Carcinomas in bold.

Visual evaluation of the PET/CT images clearly illustrated the differences between the included carcinomas and sarcomas. [Cu-64]-liposome PET/CT clearly delineated all carcinomas, except the mammary adenocarcinoma of dog 9, but none of the sarcomas after 24 h (Figure 2a-f). Importantly, the observed heterogeneity in tumor voxel uptake levels could be visually appreciated even in tumors displaying avid accumulation of [Cu-64]-liposomes (Figure 2c-d). PET/CT images of all 11 dogs are shown in Supplementary Figure S1 and S2. The observed difference in accumulation levels of [Cu-64]-liposomes between carcinomas and sarcomas are illustrated by the tumor maximum to blood mean and tumor mean to blood mean ratios, as well as tumor to liver and muscle ratios (Figure 3). The tumor-to-reference tissue ratios identify differences between tumors and underline the variation in tumor targeting potential of liposomal systems for different malignancies. The tumor-to-blood ratios between the 1-hour and 24-hour time point gives information about the extravasation of liposomes into tumor tissue. Most noteworthy is that we observe an increase in the tumor max to blood mean ratio between the 1-hour and 24-hour scans in all except one of the carcinomas but only see such an increase in one of the sarcomas (Figure 3a). Also, we only observe a very high increase in tumor mean to blood mean ratio in 2 of the dogs investigated (Figure $3 b$ ), which underlines the heterogeneity in the EPR-effect in the investigated tumor types. 

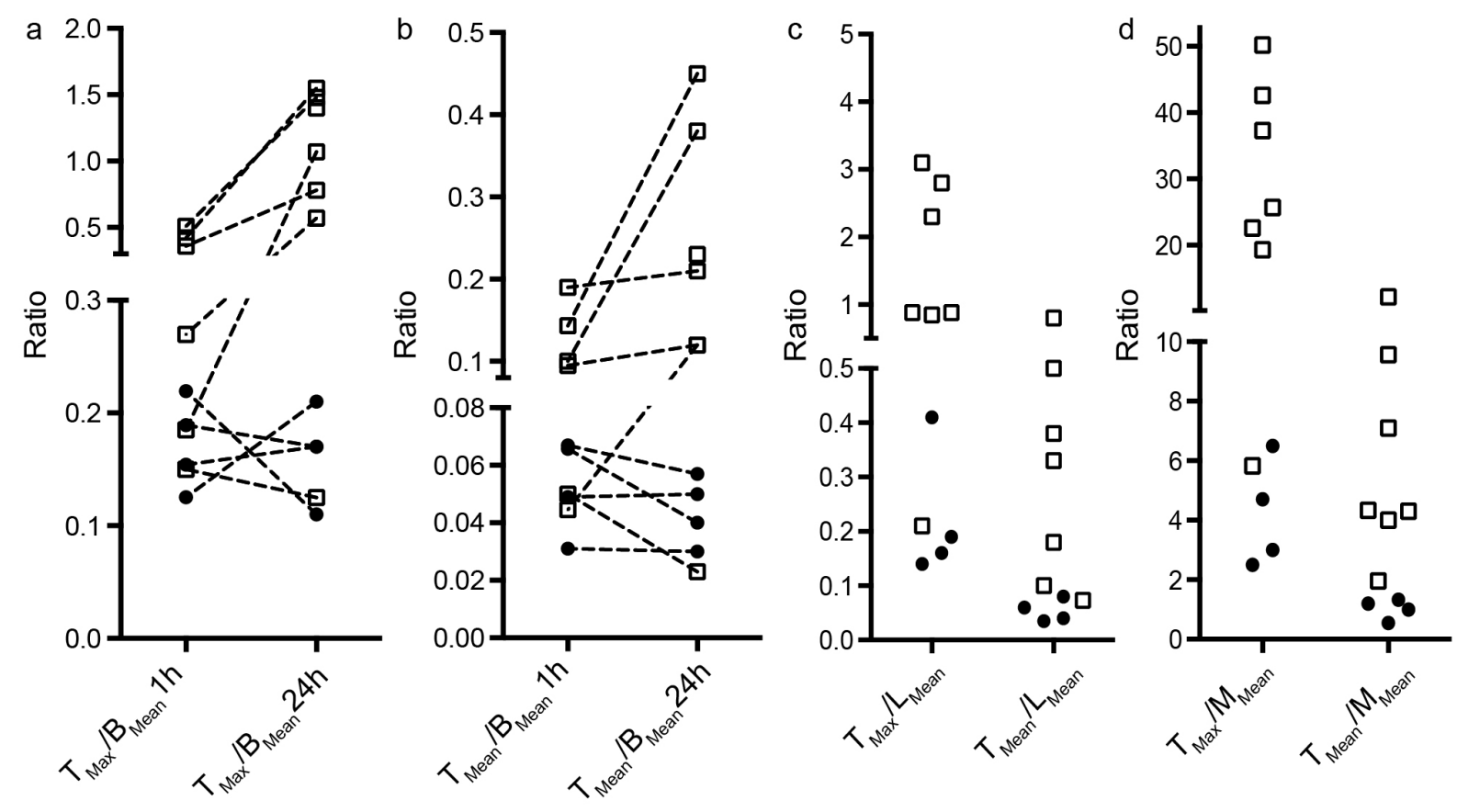

Figure 3. Tumor-to-reference tissue activity ratios. Tumor maximum to blood mean $\left(\mathrm{T}_{\max } / \mathrm{B}_{\text {mean }}\right)$ and tumor mean to blood mean ( $\left.\mathrm{T}_{\text {mean }} / \mathrm{B}_{\text {mean }}\right)$ activity ratios on the 1-hour and 24-hour PET scans (a and $\left.\mathbf{b}\right)$ and tumor maximum to liver mean $\left(\mathrm{T}_{\text {max }} / \mathrm{L}_{\text {mean }}\right)$ and tumor mean activity to liver mean $\left(\mathrm{T}_{\text {mean }} / \mathrm{L}_{\text {mean }}\right)(\mathbf{c})$ and muscle mean $\left(\mathrm{M}_{\text {mean }}\right)(\mathbf{d})$ activity ratios at the 24-hour PET scan. Carcinomas ( $\left.\square\right)$ and $\operatorname{sarcomas}(\bullet)$.

We generated histograms of voxel $\% \mathrm{ID} / \mathrm{kg}$ to provide an analysis of the voxel activity distribution (Supplementary Figure S3). From the histograms the heterogeneity in [Cu-64]liposome activity can be readily appreciated for all tumors. In order to obtain acceptable lesion identification a lesion to background ratio (e.g. tumor-to-muscle ratio) above 1.5 is generally required. ${ }^{26}$ All of the included tumors displayed a $\mathrm{T}_{\max } / \mathrm{M}_{\text {mean }}$ ratio above 1.5. However, for the sarcomas less than $30 \%$ of tumor voxels displayed an acceptable ratio whereas the majority of voxels displayed a $T_{\text {voxel }} / \mathrm{M}_{\text {mean }}$ ratio above 1.5 for the included carcinomas, except $\operatorname{dog} 9$ (Supplementary Figure S4).

We identified multiple pulmonary and lymph node metastases on the FDG PET/CT scans from $\operatorname{dog} 2$ and these displayed [Cu-64]-liposome activity (Figure 4a-b). The lymph node metastases were confirmed by cytology. FDG uptake was observed in the metastatic lesions (mean SUV $3.5-$ 6.8 and maximum SUV 5.3 - 16.7). Mean 24-hour [Cu-64]-liposome SUV for metastatic lesions ranged from $0.9-2.1$ and maximum SUV from $1.3-7.9$. These observations suggest that the EPReffect may also exist in metastases originating from primary EPR-positive tumors, thus suggesting 
that at least some macroscopic metastases will benefit from nanocarrier based drug delivery systems. No metastases were identified in any of the remaining dogs.

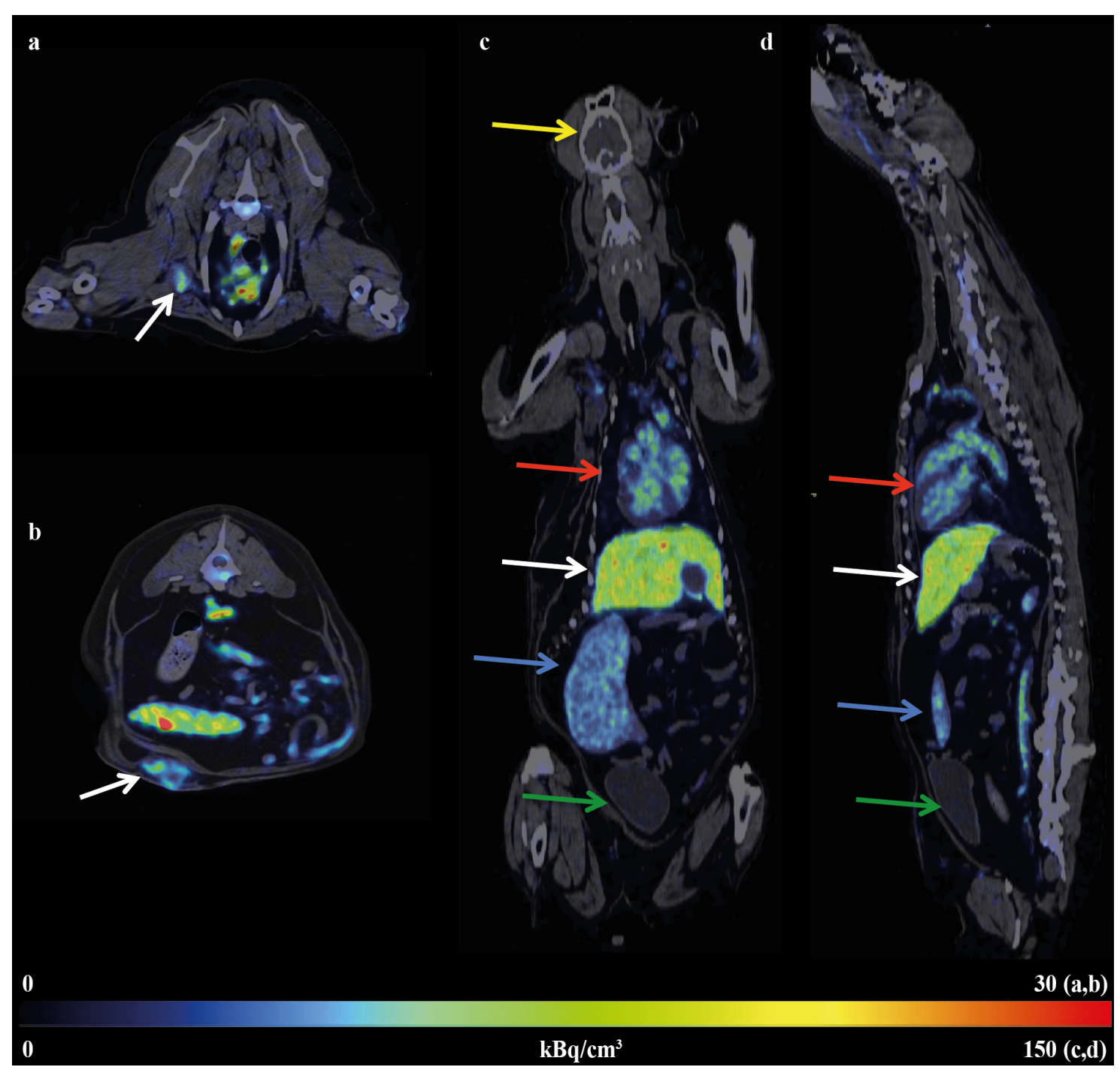

Figure 4. Transversal, coronal and sagittal [Cu-64]-liposome PET/CT images after a distribution period of $24 \mathrm{~h}$ in dog 2. (a) Transversal PET/CT image, [Cu-64]-liposome uptake can be appreciated in the axillary lymph node (arrows) and in the larger centrally located blood vessels, (b) transversal PET/CT image, [Cu64]-liposome uptake can be appreciated in the tumor (arrows), larger blood vessels and in the spleen located above the tumor. The high [Cu-64]-liposome activity levels in the blood can be appreciated in the heart ventricles, larger blood vessels, liver and spleen visualized in the coronal plane (c) and sagittal plane (d). Brain (yellow arrow), heart displaying the difference between blood activity in heart chambers and heart musculature (red arrow), liver (white arrow), where the circular gall bladder can be appreciated as a circular structure within the liver without any noteworthy [Cu-64]-liposome uptake, spleen (blue arrow) and urinary bladder (green arrow). 
Kinetics and biodistribution. We derived time activity curves (TAC) for blood, liver and spleen from the dynamic PET scan acquired during the initial 40 min of liposome distribution (including the infusion period), reconstructed at 1-minute intervals, and from the 24-hour PET scan (Figure 5a). The $\% \mathrm{ID} / \mathrm{cm}^{3}$ increases in the blood during the 20 -minute infusion period and decreases to reach a mean of $0.039 \% \mathrm{ID} / \mathrm{cm}^{3}$ (SEM 0.014) after a distribution period of $24 \mathrm{~h}$ (Figure 5a-b). The $\% \mathrm{ID} / \mathrm{cm}^{3}$ in the liver and spleen increased during the infusion and reached mean uptakes of 0.025 ID $\% / \mathrm{cm}^{3}$ (SEM 0.007) and $0.014 \mathrm{ID} \% / \mathrm{cm}^{3}$ (SEM 0.005) after $24 \mathrm{~h}$. Considering the blood volume in liver and spleen, the non-blood associated [Cu-64]-liposome activity in these organs increases over the $24 \mathrm{~h}$ period. This indicates that the PEGylated liposomes undergo the expected elimination by the reticuloendothelial system. We observed no signs of liposome leakage of Cu-DOTA on the PET images, i.e. we observed no increase in activity in the urinary bladder, we observed normal spleen or liver uptake (the standard liposome clearance organs) and the PET image analysis conveyed what could be expected for long-circulating liposomes. Comparison of [Cu-64]liposomes, free [Cu-64]-DOTA and free [Cu-64(aq)] was performed in mice to determine differences in biodistribution and accumulation kinetics (Supplementary Figure S5). Kinetics and biodistribution of the three [Cu-64] compositions indicate that [Cu-64]-liposomes are stable in dogs, i.e. no indication of leakage of [Cu-64]-DOTA was observed. Additionally, a study where [Cu-64]-liposomes were incubated in canine plasma revealed that the stability of [Cu-64]-liposomes was very high with $<3 \%$ leakage of [Cu-64]-DOTA (Supplementary Figure S6).
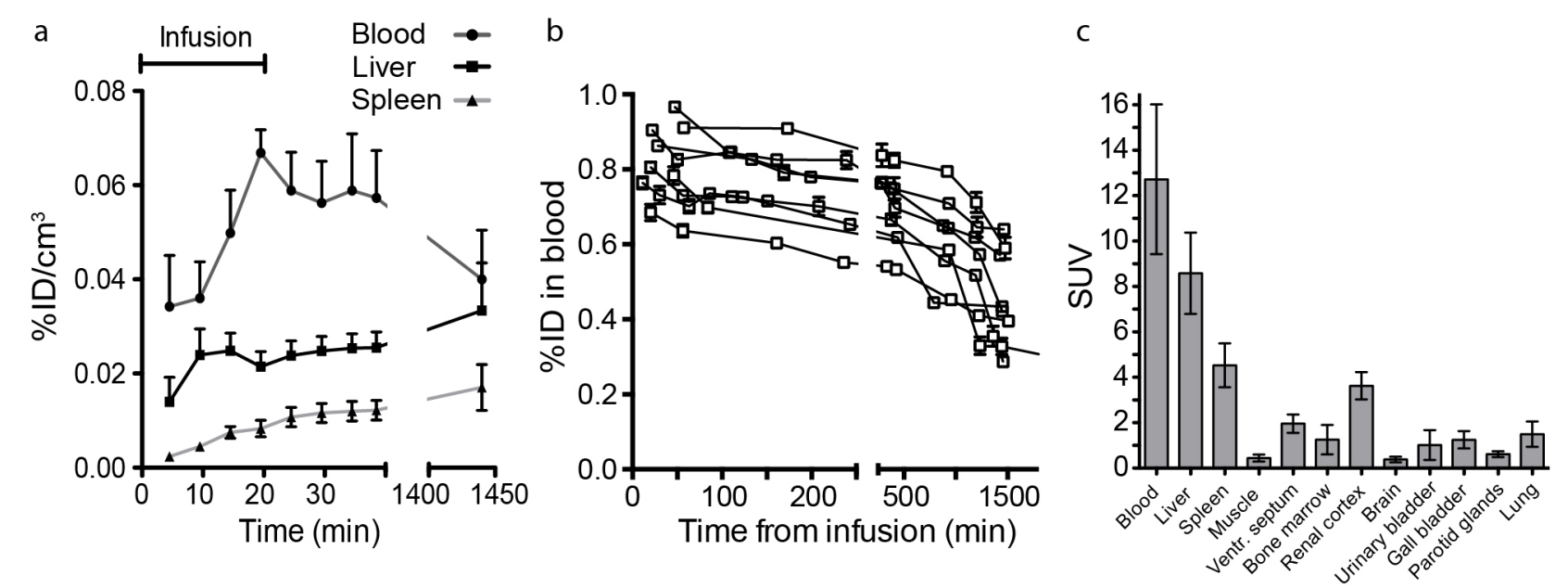

Figure 5. [Cu-64]-liposome distribution and circulating properties. (a) Percentage of injected dose (\%ID) of [Cu-64]-liposomes pr. $\mathrm{cm}^{3}$ of blood, liver and spleen plotted against distribution time for the initial $40 \mathrm{~min}$ 
and after approximately $24 \mathrm{~h}$ (mean \pm SEM). (b) Time activity curves (TAC) of fraction of injected activity of [Cu-64]-liposomes in blood circulation at various distribution time points. Fraction of injected activity is based on a calculated blood volume of $8 \%$ of total bodyweight (mean \pm SEM). (c) Biodistribution of [Cu64]-liposomes after a distribution period of approximately $24 \mathrm{~h}$ (standardized uptake value (SUV) mean \pm SEM).

TACs of decay corrected percentage of [Cu-64]-liposome in circulation were calculated from triplicates of well-counted blood samples (Figure 5b). Circulating half-life was estimated to $35.0 \mathrm{~h}$ (SEM $4.2 \mathrm{~h}$ ), based on non-linear one-phase exponential decay. Goodness-of-fit $\left(\mathrm{R}^{2}\right)$ ranged from $0.80-0.92$ indicating an acceptable curve fit. The estimated circulating half-life is comparable to those reported in humans for pegylated liposomal doxorubicin (Doxil ${ }^{\mathrm{TM}}$ ) and [In-111]-labeled pegylated liposomes. ${ }^{15,27}$ The circulating half-life of the liposome formulation could be appreciated on the 24-hour PET/CT images (Figure 4).

Biodistribution in selected tissues and organs was determined from the 24-hour [Cu-64]liposome PET scans (Figure 5c). High activity of [Cu-64]-liposomes was observed in the aorta, liver, spleen and renal cortices, whereas the lowest activity was observed in bone marrow (proximal humerus), lung, muscle, brain and parotid glands.

\section{Discussion}

In the present study we have developed a new method for preparing PET radionuclide $[\mathrm{Cu}-64]^{2+}$ labelled liposomes that can easily be used by for radiolabeling liposomes in both preclinical and clinical settings. The highly efficient and fast loading procedure that was developed, where $[\mathrm{Cu}-$ $64]^{2+}$ is efficiently diffusing across the liposome membrane at elevated temperature was a surprise to us and we tested loading of $\mathrm{Ca}^{2+}$ to investigate if this was a general phenomenon. $\mathrm{Ca}^{2+}$ was not able to diffuse across the liposome membrane and it seems that there are differences in the membrane interactions of $\mathrm{Cu}^{2+}$ and $\mathrm{Ca}^{2+}$. The exact mechanism that is causing this difference is unknown to us and we were highly surprised that we can load $[\mathrm{Cu}-64]^{2+}$ so effectively into liposomes by using a chemical gradient constituted by the DOTA chelate. We will in future studies further investigate this phenomenon. This study furthermore represents the first PET/CT study of a radiolabeled nanocarrier in a clinical animal model with spontaneous tumors. The PET/CT imaging provided high-resolution and quantitative data on liposome tumor accumulation in dogs with cancer, which are comparable to human cancers. Thus, the study provides unique new information that should be evaluated in the context of how nanocarrier based drug delivery systems of antitumor 
agents that depends on the EPR-effect will improve intratumoral accumulation and therapeutic efficacy relative to corresponding free drugs. Additionally, uptake and release kinetics must be appropriate relative to the drug delivered in order to achieve maximal efficacy. Based on these considerations tumor targeted nanocarrier drug delivery systems may prove to have very different efficacy for different tumors and could potentially decrease the therapeutic efficacy relative to the free-agent in some instances. ${ }^{28}$ The liposome formulation under investigation is the same as used in the marketed formulation Doxil with respect to liposome size and lipid composition but does not have the elongated shape as Doxil. The data reported on tumor uptake and intratumoral heterogeneity is therefore expected to be representative for long-circulating liposome formulations and potentially also a number of other PEGylated nanocarriers in the same size range.

Radiolabeled liposomes have previously been evaluated in human cancer patients. ${ }^{15-18}$ The tumor accumulation levels of [Cu-64]-liposomes in the included carcinomas that displayed EPR effects (based on increasing accumulation between scans) are compatible to those reported in previous human cancers evaluated by gamma camera and SPECT imaging. ${ }^{15}$ The authors report tumor uptake from tumor size estimations to range from $2.7-53.0 \% \mathrm{ID} / \mathrm{kg}$. Tumor uptake was found to decrease with increasing tumor size and highest uptake was observed in head and neck squamous cell carcinoma (SCC), intermediate uptake in lung cancers and lowest in breast cancers. ${ }^{15}$ However, these reports in human cancers have only provided semi-quantitative estimation of liposome biodistribution based on single photon imaging at a single time-point, which, considering circulation times and tumor blood volume, limits the evaluation of the EPR-effect. Due to the high detection sensitivity and spatial resolution and the true quantitative nature of PET we can report accurate 3D quantitative measurements of [Cu-64]-liposome distribution after a shorter distribution period. Tumor-to-blood ratios are therefore reported at $1 \mathrm{~h}$ and $24 \mathrm{~h}$ after liposome infusion. This analysis clearly demonstrates the EPR-effect, as the tumor-to-blood ratio will increase if the nanocarriers extravasate into the interstitial space in the tumor. The EPR-effect was found to be present only in the carcinomas, except one mammary adenocarcinoma, as derived from the increased uptake level and retention of liposomes after a distribution period of $24 \mathrm{~h}$ (evaluated by tumor-to-blood ratios). The small SCC of dog 1 displayed the largest increase in \% ID/kg and tumor-to-muscle ratio between $1 \mathrm{~h}$ and $24 \mathrm{~h}$ of distribution, which is in accordance with previous reports. ${ }^{15,29}$ The study by Harrington et al ${ }^{15}$ reports four of five breast cancers as positive (visible) on both planar gamma camera and SPECT imaging after $72 \mathrm{~h}$. However, activity levels of radiolabeled liposomes in at least two, but potentially 3 out of 4 , of the positive breast cancers are 
comparable to those observed in the present study after only $1 \mathrm{~h}$ of liposome distribution and it may therefore be more reflective of tumor blood volume. This observation questions whether positive images always reflect the EPR-effect and underlines that a minimum of two scanning time points may be needed to identify the EPR-effect by imaging. Due to the limited number of studies and the potential poor correlation between tumor visualization by imaging and the presence of the EPReffect in tumors, further studies are warranted in humans to clarify which tumor types that can benefit the most from nanocarrier based therapeutics.

The observed intratumoral heterogeneity in liposome accumulation underlines the importance of further elucidation of liposome accumulation and drug release. To what extent this is influenced by hypoxic regions, interstitial fluid pressure and regional differences in vasoactive factors or how it will influence distribution of liposomes functionalized with ligands that target tumor specific receptors is not clear and studies are required to identify the interdependence of these parameters. This study therefore, in addition to providing new information on EPR-based tumor accumulation and heterogeneity, also highlights the importance of further elucidating the drug distribution of liposome-encapsulated drug following their release. These issues may be a potential Achilles heel for nanocarrier based drug delivery systems as cancer cells with known therapeutic resistance, including hypoxic cells and cancer stem cells, may reside in perinecrotic and/or hypoxic regions and niches displaying low accumulation of nanocarriers. When evaluating the histograms and activity levels of this study the resolution of the PET scan and reconstruction algorithm must be taken into consideration as each voxel represent a large number of cancer cells and a small, potentially heterogeneous, tumor sub-volume. The histograms therefore provide information on the liposome concentrations that can be achieved within solid tumors, which again must be compared to studies of drug release kinetics and diffusion and distribution of released free anti-tumor agents.

The accumulation level (\%ID) that was achieved in the tumors in this study, relative to those reported for experimental models, may if translated directly, seem disappointing. However, we have previously reported tumor accumulation levels of passively accumulating radiolabeled liposomes to range from 4.8 to $6.0 \% \mathrm{ID} / \mathrm{cm}^{3}$ between 24 and $48 \mathrm{~h}$ after injection in different xenograft models. ${ }^{24,30}$ However, if considering that the tumors of the experimental animal models represented between 2 $5 \%$ of the total bodyweight and applying a similar relationship between tumor and host compatible for the carcinomas of the present dog study similar numbers are achieved based on $\%$ ID $/ \mathrm{cm}^{3}$. The evaluation of liposomal accumulation in experimental rodent models must therefore be carefully interpreted when investigating the potential for imaging and drug delivery systems. 
Based on the findings in the present study, EPR-based passive accumulation of PEGylated liposomes (or other nanocarriers) in the $100 \mathrm{~nm}$ size-range cannot be considered a universal feature of all solid tumors. In this study, the SCC tumor had the highest EPR-effect and it is noteworthy that the majority of tumors included in the Harrington et al. ${ }^{15}$ study also were SCC tumors. Different approaches are currently being investigated to improve and increase the EPR-effect, including downsizing of nanoparticles and manipulation of tumor microenvironment, ${ }^{10,31}$ which could generate important information that could expand the tumor types where nanocarriers will be effective. Another approach has been to specifically trigger drug release from nanocarriers within the tumor vasculature to improve drug distribution in tumor. ${ }^{32}$ Furthermore, the influence of cytotoxic agents, anti-angiogenic drugs and radiotherapy must be investigated; to identify their impact on EPR-based drug delivery. Importantly, the optimization of drug delivery may become central in cancer therapy when considering the growing skepticism towards the possibilities and perspectives for individualized targeted therapy, ${ }^{33}$ making tumor specific and dose escalating delivery of chemotherapeutic agents an attractive methodology to overcome resistance and improve treatment outcome.

\section{Conclusion}

In the present article we have presented a new and highly efficient method for loading copper-64 PET isotopes into liposomes without the use of ionophores based on a highly surprising diffusion of copper ions across liposome membranes where it is then entrapped by a high affinity copper chelator. This new loading method will be highly valuable in future PET studies with liposomes in animals and patients. Furthermore, even with the moderate number of 11 canine cancer patients and different histologies included, which is a consequence of the logistics of using family owned canine patients, this study identifies several highly important issues for nanocarrier based drug delivery to spontaneous tumors, including tumor type dependent differences and accumulation heterogeneity. Moderate to high nanocarrier tumor accumulation levels were achieved in spontaneous carcinomas based on the EPR-effect, which underlines the potential of nanocarrier drug delivery systems for elevating drug concentrations specifically within certain tumors. However, heterogeneity in intratumoral liposome distribution and drug release in the extracellular matrix may prove to be very important issues for optimization of liposomal drug delivery to achieve high therapeutic efficacy. Importantly, based on our observations, studies of specific malignancies and potentially even tumors of individual patients are needed to identify patients that are likely to benefit from 
nanocarrier based drug delivery. Radiolabeled liposomes may therefore serve as theranostic imaging agent guiding both diagnostic and therapeutic intervention for several malignancies in future clinical practice and guide patient selection for optimized clinical trails.

\section{Materials and Methods}

Study population. Canine cancer patients suffering from solid malignant tumors were eligible for inclusion. All dogs underwent clinical examination and had blood biochemical, hematological and urine analysis performed in preparation for anesthesia and to identify concurrent illnesses. Owners of included dogs provided written informed consent prior to inclusion. Eleven dogs met the inclusion criteria; 5 soft tissue sarcomas, 6 carcinomas (Table 1). The study protocol was approved by the ethical and administrative committee at the Department of Small Animal Clinical Sciences, University of Copenhagen, Denmark.

Liposome formulation and production - Materials. Freeze-dried lipid mixture, hydrogenated soy phosphatidylcholine (HSPC)/cholesterol (Chol)/1,2-distearoyl-sn-glycero-3-phosphoethanolamine$N$-[methoxy (polyethylene glycol)-2000] $\left(\right.$ DSPE-PEG $_{2000}$ ) in the molar ratio (56.5:38.2:5.3) was purchased from Lipoid ${ }^{\circledR} \mathrm{GmbH}$ (Ludwigshafen, Germany). 1,4,7,10-tetra-azacyclododecane1,4,7,10-tetraacetic acid (DOTA) was purchased from Macrocyclics (Dallas, USA). All other solvents and chemicals were purchased from Sigma Aldrich (Schnelldorf, Germany) and were used without further purification. $10 \mathrm{ml}$ LIPEX Thermobarrel Extruder and polycarbonate membranes were purchased from Northern Lipids (Burnaby, Canada) and the Minimate ${ }^{\mathrm{TM}}$ tangential flow filtration system was purchased from Pall Corporation (Canada).

Preparation of DOTA-liposomes and Empty-liposomes. Freeze-dried Lipoid ${ }^{\circledR}$ lipid mixture: HSPC/Chol/DSPE-PEG ${ }_{2000}$ in the molar ratio (56.5:38.2:5.3) was hydrated with a HEPES buffer (10 $\mathrm{mM}, 150 \mathrm{mM} \mathrm{NaCl}, \mathrm{pH} 7.4)$ containing DOTA $\left(\mathrm{C}_{\text {ротА }}=10 \mathrm{mM}\right)$ adjusted to $\mathrm{pH} 7.4$ and 335 $\mathrm{mOsm} / 1$, and subsequently placed on a heating block with stirring at $65^{\circ} \mathrm{C}$ for $80 \mathrm{~min}$. The hydrated solution was then extruded through polycarbonate membranes with pore sizes of $100 \mathrm{~nm}$ to generate $100 \mathrm{~nm}$ sized liposomes using a $10 \mathrm{ml}$ LIPEX Thermobarrel Extruder placed on a heating block at $65^{\circ} \mathrm{C}$ during the extrusion process. The extruded $100 \mathrm{~nm}$ liposome-solution was then transferred to a Minimate ${ }^{\mathrm{TM}}$ tangential flow filtration system to remove un-encapsulated DOTA on the outside of the liposomes. The external buffer solution containing DOTA was replaced with a 
HEPES buffer (10 mM, $150 \mathrm{mM} \mathrm{NaCl}, \mathrm{pH}$ 7.4, $295 \mathrm{mOsm} / \mathrm{l})$. The purified DOTA-containing liposomes were then loaded with [Cu-64] (as described below) before in vivo administration.

Empty-liposomes consisting of freeze-dried Lipoid ${ }^{\circledR}$ lipid mixture: HSPC/Chol/DSPE-PEG ${ }_{2000}$ in the molar ratio (56.5:38.2:5.3) were prepared as follow. The lipids were dispersed by adding an aqueous solution, a HEPES buffer (10 mM, $150 \mathrm{mM} \mathrm{NaCl}, \mathrm{pH} \mathrm{7.4,} 295 \mathrm{mOsm} / \mathrm{L})$. The solution was then hydrated at $65^{\circ} \mathrm{C}$ for 60 min followed by extrusion through polycarbonate membranes to generate $100 \mathrm{~nm}$ sized liposomes using a $10 \mathrm{ml}$ LIPEX Thermobarrel Extruder.

The size and zeta-potential of both DOTA-liposomes and Empty-liposomes were monitored using dynamic light scattering (ZetaPALS, Brookhaven Instruments, US) over the period where dogs were enrolled in the study, which yielded an average diameter of $110 \mathrm{~nm} \pm 5 \mathrm{~nm}$ (PDI < $0.075)$ and an average zeta-potential of $-7 \mathrm{mV} \pm 3 \mathrm{mV}$. The lipid/phosphorus concentration was verified by measurement of phosphorus concentration using ICP-MS (iCAP Q, Thermo Scientific, DE) using Gallium as internal standard.

[Cu-64] production. Copper-64 was produced on a PETtrace cyclotron (GE Healthcare) equipped with a beamline. The production of [Cu-64] was carried out via the ${ }^{64} \mathrm{Ni}(\mathrm{p}, \mathrm{n}){ }^{64} \mathrm{Cu}$ nuclear reaction using a solid target system consisting of a water cooled target mounted on the beamline. The target consisted of [Ni-64] metal (enriched to $>99 \%$ ) electroplated on a silver disc backing. A proton beam of $16 \mathrm{MeV}$ and a beam current of $20 \mathrm{~mA}$ were used. After irradiation, the target was transferred to the laboratory for further chemical processing where [Cu-64] was isolated using ion exchange chromatography. Final evaporation from aqueous $\mathrm{HCl}$ yielded $[\mathrm{Cu}-64]$ as $[\mathrm{Cu}-64] \mathrm{Cl}_{2}$.

[Cu-64] labeled liposomes. Purified DOTA-containing liposomes were added to the vial containing the radioactive $[\mathrm{Cu}-64] \mathrm{Cl}_{2}$ followed by incubation at $50-55^{\circ} \mathrm{C}$ for $60 \mathrm{~min}$. The fraction of un-entrapped [Cu-64]-DOTA in the loaded [Cu-64]-liposome solution was quantified by separating [Cu-64]-DOTA from [Cu-64]-liposomes by size exclusion chromatography using a Sephadex G-50 column (1x25 cm) eluted with a HEPES buffer (10 mM, $150 \mathrm{mM} \mathrm{NaCl}, \mathrm{pH} 7.4)$. The fraction of un-entrapped free [Cu-64] in the [Cu-64]-liposome solution was quantified by separating un-entrapped [Cu-64] from [Cu-64]-DOTA by radio-thin layer chromatography (radioTLC). The radiochemical purity of the produced radiolabeled [Cu-64]-liposomes was $>95 \%$ in all productions used in this study. Before infusion, Empty-liposomes (4.3 mg lipid/kg canine) were 
added to the [Cu-64]-liposomes solution to ensure injection of a sufficient amount of liposomes to make them long circulating.

PET/CT. PET/CT scans were performed using a combined PET/CT scanner (Biograph 40 PET/CT or Biograph 64 PET/CT, Siemens, Erlangen, Germany); consisting of a high resolution PET scanner (4 x 4 mm LSO crystal elements, 32,440 LSO crystals, $21.6 \mathrm{~cm}$ axial field) and a 40 or 64-row multi-slice CT scanner. CT parameters used were a slice thickness of $3.0 \mathrm{~mm}, 120 \mathrm{kV}, 170$ mAs, pitch 1.2, collimation $24 \times 1.2 \mathrm{~mm}$ and a B30 kernel. The PET scans were acquired using static image acquisition and dynamic list mode acquisition. Images were reconstructed using a 3D acquisition mode and attenuation corrected using the concurrent CT scan. PET images were reconstructed using TrueX ${ }^{\circledR}$ (Siemens, Erlangen, Germany) 3D reconstruction (21 iterations, 3 subsets), and smoothed using a Gaussian filter having a FWHM of $2 \mathrm{~mm}$ in all directions, and a matrix size of $336 \times 336$.

Study procedure FDG PET/CT (day 1). The dogs were fasted for a minimum of $12 \mathrm{~h}$ prior to imaging and all were confirmed to have normal serum glucose concentrations. Dogs were premedicated with Methadone ( 0.2 to $0.3 \mathrm{mg} / \mathrm{kg} \mathrm{IM})$. FDG was injected intravenously as a bolus. Dogs received a mean FDG activity of 7.0 MBq/kg (range: 3.8-9.85 MBq/kg).

All dogs were visually monitored after FDG administration. Following a distribution period for the tracer (mean $61 \mathrm{~min}$ (range: 56-67 min)), anaesthesia was performed using a continuous infusion of propofol $(15-25 \mathrm{mg} / \mathrm{kg} / \mathrm{h})$, and provided with $100 \%$ oxygen via a tracheal tube. FDG PET/CT scans were performed as a 3-minute multi field of view (FOV) whole body PET scan.

Study procedure [Cu-64]-liposome PET/CT (day 2 and 3). [Cu-64]-liposome PET scans were performed as two separate scanning session commencing between 24 and $48 \mathrm{~h}$ after the FDG PET/CT scan. The first [Cu-64]-liposome scan was initiated simultaneously with the infusion of radiolabeled liposomes (termed day 1-hour scan) and the second liposome scan was performed approximately $24 \mathrm{~h}$ after (termed day 24-hour scan). Anesthesia was conducted as for the FDG PET/CT scan and all dogs received $2 \mathrm{mg} / \mathrm{kg}$ dexamethasone disodium phosphate $\sim 2 \mathrm{~h}$ prior to infusion of [Cu-64]-liposomes to minimize risks for immunogenic reactions. Radiolabeled liposomes were infused at increasing rates over a period of $20 \mathrm{~min}$ using an automated injection pump. Infusion rates were; $0.5 \mathrm{ml} / \mathrm{min}(0-5 \mathrm{~min}), 1.0 \mathrm{ml} / \mathrm{min}(5-10 \mathrm{~min}), 2.5 \mathrm{ml} / \mathrm{min}(10-15 \mathrm{~min})$ 
and $4 \mathrm{ml} / \mathrm{min}(15-20 \mathrm{~min})$ for a total infusion volume of $40 \mathrm{ml}$. Dogs received a mean [Cu-64]liposomal activity of $\sim 10 \mathrm{MBq} / \mathrm{kg}$.

The 1-hour [Cu-64]-liposome PET/CT scan was performed as a dynamic list mode acquisition. In dogs 1 to 6 the PET scanner FOV was positioned to cover the liver and spleen region for the initial 0-40 min. After $40 \mathrm{~min}$ the PET scanner FOV was moved to the tumor region for a 20 minute dynamic list mode acquisition. Dynamic list mode data were temporally reconstructed into images at $1 \mathrm{~min}$ intervals and the list mode data from the tumor region was additionally reconstructed into a summarized 5-minute image based on 55-60 min list mode data. The tumor of dog 2 could not be covered by a single FOV and an additional static 2.5-minute FOV scan was performed to cover the entire tumor volume and these images served as the basis for determining tumor uptake values at the 1-hour scans.

The 24-hour [Cu-64]-liposome PET/CT scan was initiated by a static 5 min FOV acquisition in the tumor region followed by a 2.5 min FOV whole body PET scan. [Cu-64]-liposome PET/CT scan were reconstructed using the same TrueX ${ }^{\circledR}$ reconstruction algorithm and parameters as for the FDG PET scans and attenuation corrected using the concurrently acquired CT scan.

Image analysis. Attenuation corrected and reconstructed PET/CT images were post processed using commercial software (Pmod, ver. 3.304, Pmod Technologies, Switzerland).

Tumor volumes of interests (VOIs) were manually delineated on the FDG PET/CT and [Cu-64]liposome PET/CT scans, without applying any threshold for the PET uptake following the different malignancies being evaluated. FDG uptake was reported as tumor mean and maximum standardized uptake value (SUV). Tumor uptake of [Cu-64]-liposome on the 1-hour (5-minute reconstruction, 55-60 min list mode data) and 24-hour tumor scans were reported as tumor mean and maximum SUV and 24-hour scan voxel uptake histogram constructed. Tumor uptake percentage of injected dose (\%ID) was calculated for the whole tumor and for voxel mean and maximum. Intratumoral heterogeneity of liposome accumulation was investigated by constructing voxel uptake histograms of tumor VOIs based on the 24-hour PET images.

Pulmonary and lymph node metastases were identified and delineated in dog 2, the uptake levels of FDG and [Cu-64]-liposome are reported as for the primary tumors.

PET time activity curves (PET-TAC) for blood, liver and spleen were generated from the 1-hour scan on temporally reconstructed images. Blood activity was determined by generating a large VOI around the aorta and subsequently segmenting the VOI by an $80 \%$ of maximum threshold to 
minimize partial volume effects and non-blood associated activity. VOIs manually drawn well within the margins of liver and spleen on five consecutive image slices were used for the determination of temporal activity. PET Blood activity for the determination of tumor to blood ratios at the 1-hour PET scan was estimated by decay correcting average blood activity 38-40 min post-injection (pi) to the 1-hour time point of tumor activity. For technical reasons TAC curves were not constructed for dogs 9 and 10.

[Cu-64]-liposome biodistribution and tumor to reference tissue uptake ratios at the 24-hour whole body PET scan $24 \mathrm{~h}$ after liposome infusion were evaluated by constructing reference VOIs in the following tissues; aorta, liver, spleen, muscle (average of five regions), lung (peripheral region), bone marrow (proximal humerus, representing red bone marrow), ventricular septum, parotid salivary glands, urinary bladder and gall bladder. All regions were drawn well within the margins of tissues and organs and excluding regions containing larger blood vessels, e.g. the hilar region of the liver, to avoid artifacts and minimize partial volume effects and respiratory movement. Aorta VOIs were constructed as described above. Ventricular septum VOIs was formed by PET uptake based on segmentation to minimize the effects of blood activity in heart ventricles and heart movement during scanning. In short a spherical VOI including the right and left heart ventricles and ventricular septum was constructed and segmentation performed.

Time activity curves (TACs). TACs were generated for the individual dogs by collecting multiple blood samples. Blood samples were collected as allowed for during and after the [Cu-64]liposome PET/CT scans without interfering with scanning procedures. Ten dogs had blood samples collected between 11 to 1509 min after completing the liposome infusion. EDTA stabilized blood samples were well counted (Gamma counter, Perkin Elmer, Australia) in triplicates and decay corrected specific blood activity of [Cu-64]-liposome calculated. The relative percentage of $[\mathrm{Cu}-$ 64]-liposome in circulation was estimated from an expected blood mass of $8 \%$ of total body weight and relative blood content plotted against distribution period. Circulating half-life of liposomes was calculated using the non-linear one-phase exponential decay equation: $y=\mathrm{Ae}^{-\mathrm{kt}}, \mathrm{t}_{1 / 2}=0.693 / \mathrm{k}$.

Acknowledgements. Financial support was kindly provided by the Danish Strategic Research Council (NABIIT) Ref. 2106-07-0033, The Lundbeck Foundation (Fellowship program) and the European Research Council (ERC grant). The funding sources were not involved or influenced the design and execution of the study or publication in any way. 
Supporting Information Available. PET images of all 11 dogs (Figure S1 and S2), Histograms displaying voxel-wise uptake characteristics of $\% \mathrm{ID} / \mathrm{kg}[\mathrm{Cu}-64]$-liposome in tumor from included dogs (Figure S3), Histograms displaying voxel-wise tumor $_{\text {voxel }}$-to-muscle $_{\text {mean }}$ ratios [Cu-64]liposome activity (Figure S4), PET/CT images of [64-Cu(aq)], [Cu-64]-DOTA and [Cu-64]liposomes in mice (Figure S5) and In vitro stability test of liposomes loaded with ${ }^{64} \mathrm{Cu}$ in $\operatorname{dog}$ plasma (Figure S6). This material is available free of charge via the internet at http://pubs.acs.org.

\section{References}

1. Barenholz, Y. Doxil(R)-the First FDA-approved Nano-drug: Lessons Learned. J. Controlled Release 2012, 160, 117-134.

2. Hrkach, J.; Von Hoff, D.; Ali, M. M.; Andrianova, E.; Auer J.; Campbell, T.; De Witt, D.; Figa, M.; Figueiredo, M.; Horhota, A.; et al. Preclinical Development and Clinical Translation of a PSMA-Targeted Docetaxel Nanoparticle with a Differentiated Pharmacological Profile. Sci. Transl. Med. 2012, 4, 128ra39.

3. Matsumura, Y.; Maeda, H. A New Concept for Macromolecular Therapeutics in Cancer Chemotherapy: Mechanism of Tumoritropic Accumulation of Proteins and the Antitumor Agent Smancs. Cancer Res. 1986, 46, 6387-6392.

4. Bangham, A. D. Surrogate Cells or Trojan Horses. The Discovery of Liposomes. BioEssays 1995, 17, 1081-1088.

5. Iyer, A. K.; Khaled, G.; Fang, J.; Maeda, H. Exploiting the Enhanced Permeability and Retention Effect for Tumor Targeting. Drug Discovery Today 2006, 11, 812-818.

6. Zhang, L.; Gu, F. X.; Chan, J. M.; Wang, A. Z.; Langer, R. S.; Farokhzad, O. C. Nanoparticles in Medicine: Therapeutic Applications and Developments. Clin. Pharm. Ther. 2008, 83, 761-769.

7. Folkman, J. Fundamental Concepts of the Angiogenic Process. Curr. Mol. Medicine 2003, 3, 643-651.

8. Hanahan, D.; Weinberg, R. A. Hallmarks of Cancer: The Next Generation. Cell 2011, 144, 646674. 
9. Maeda, H.; Bharate, G. Y.; Daruwalla, J. Polymeric Drugs for Efficient Tumor-Targeted Drug Delivery based on EPR-effect. Eur. J. Pharm. Biopharm. 2009, 71, 409-419.

10. Fang, J.; Nakamura, H.; Maeda, H. The EPR effect: Unique Features of Tumor Blood Vessels for Drug Delivery, Factors Involved, and Limitations and Augmentation of the Effect. Adv. Drug Deliv. Rev. 2011, 63, 136-151.

11. Denison, T. A.; Bae, Y. H. Tumor Heterogeneity and its Implication for Drug Delivery. $J$. Controlled Release 2012, 164, 187-191.

12. Connolly, D. T.; Heuvelman, D. M.; Nelson, R.; Olander, J. V.; Eppley, B. L.; Delfino, J. J.; Siegel, N. R.; Leimgruber, R. M.; Feder, J. Tumor Vascular Permeability Factor Stimulates Endothelial Cell Growth and Angiogenesis. J. Clin. Invest. 1989, 84, 1470-1478.

13. Minko, T.; Kopeckova, P.; Pozharov, V.; Jensen, K. D.; Kopecek, J. The Influence of Cytotoxicity of Macromolecules and of VEGF Gene Modulated Vascular Permeability on the Enhanced Permeability and Retention Effect in Resistant Solid Tumors. Pharm. Res. 2000, 17, $505-514$.

14. Safra, T. Cardiac Safety of Liposomal Anthracyclines. Oncologist 2003, 8, 17-24.

15. Harrington, K. J.; Mohammadtaghi, S.; Uster, P. S.; Glass, D.; Peters, A. M.; Vile, R. G.; Stewart, J. S. Effective Targeting of Solid Tumors in Patients with Locally Advanced Cancers by Radiolabeled Pegylated Liposomes. Clin. Cancer Res. 2001, 7, 243-254.

16. Presant, C. A.; Blayney, D.; Proffitt, R. T.; Turner, A. F.; Williams, L. E.; Nadel, H. I.; Kennedy, P.; Wiseman, C.; Gala, K.; Crossley, R. J.; et al. Preliminary report: Imaging of Kaposi Sarcoma and Lymphoma in AIDS with Indium-111-labelled Liposomes. Lancet 1990, 335, 1307-1309.

17. Khalifa, A.; Dodds, D.; Rampling, R.; Paterson, J.; Murray, T. Liposomal Distribution in Malignant Glioma: Possibilities for Therapy. Nucl. Med.Commun. 1997, 18, 17-23.

18. Presant, C. A.; Proffitt, R. T.; Turner, A. F.; Williams, L. E.; Winsor, D.; Werner, J. L.; Kennedy, P.; Wiseman, C.; Gala, K.; McKenna, R. J.; et al. Successful Imaging of Human Cancer with Indium-111-labeled Phospholipid Vesicles. Cancer 1988, 62, 905-911.

19. Paoloni, M.; Khanna, C. Translation of New Cancer Treatments from Pet Dogs to Humans. Nat. Rev. Cancer 2008, 8, 147-156.

20. Gambhir, S. S. Molecular Imaging of Cancer with Positron Emission Tomography. Nat. Rev. Cancer 2002, 2, 683-693. 
21. Phelps, M. E.; Hoffman, E. J.; Mullani, N. A.; Ter-Pogossian, M. M. Application of Annihilation Coincidence Detection to Transaxial Reconstruction Tomography. J. Nucl. Med. 1975, 16, 210-224.

22. Seo, J. W.; Qin, S.; Mahakian, L. M.; Watson, K. D.; Kheirolomoom, A.; Ferrara, K. W. Positron Emission Tomography Imaging of the Stability of Cu-64 labeled Dipalmitoyl and Distearoyl Lipids in Liposomes. J. Controlled Release 2011, 151, 28-34.

23. Willmann, J. K.; van Bruggen, N.; Dinkelborg, L. M.; Gambhir, S. S. Molecular Imaging in Drug Development. Nat.Rev. Drug Discovery 2008, 7, 591-607.

24. Petersen, A. L.; Binderup, T.; Rasmussen, P.; Henriksen, J. R.; Elema, D. R.; Kjaer, A.; Andresen, T. L. ${ }^{64} \mathrm{Cu}$ Loaded Liposomes as Positron Emission Tomography Imaging Agents. Biomaterials 2011, 32, 2334-2341.

25. Hansen, A. E.; McEvoy, F.; Engelholm, S. A.; Law, I.; Kristensen, A. T. FDG PET/CT Imaging in Canine Cancer Patients. Vet. Radiol. Ultrasound 2011, 52, 201-206.

26. Phillips, W. T. Delivery of Gamma-Imaging Agents by Liposomes. Adv. Drug Deliv. Rev. 1999, 37, 13-32.

27. Gabizon, A.; Shmeeda, H.; Barenholz, Y. Pharmacokinetics of Pegylated Liposomal Doxorubicin: Review of Animal and Human Studies. Clin. Pharmacokinet. 2003, 42, 419-436.

28. Gabizon, A. A. Stealth Liposomes and Tumor Targeting: One Step Further in the Quest for the Magic Bullet. Clin. Cancer Res. 2001, 7, 223-225.

29. Harrington, K. J.; Rowlinson-Busza, G.; Syrigos, K. N.; Abra, R. M.; Uster, P. S.; Peters, A. M.; Stewart, J. S. Influence of Tumour Size on Uptake of (111)ln-DTPA-labelled Pegylated Liposomes in a Human Tumour Xenograft Model. Br. J. Cancer 2000, 83, 684-688.

30. Petersen, A. L.; Binderup, T.; Jølck, R. I.; Rasmussen, P.; Henriksen, J. R.; Pfeifer, A. K.; Kjaer, A.; Andresen, T. L. Positron Emission Tomography Evaluation of Somatostatin Receptor Targeted ${ }^{64} \mathrm{Cu}$-TATE-Liposomes in a Human Neuroendocrine Carcinoma Mouse Model. J. Controlled Release 2012, 160, 254-263.

31. Cheng, C. J.; Saltzman, W. M. Nanomedicine: Downsizing Tumour Therapeutics. Nat. Nanotech. 2012, 7, 346-347.

32. Manzoor, A. A.; Lindner, L. H.; Landon, C. D.; Park, J. Y.; Simnick, A. J.; Dreher, M. R.; Das, S.; Hanna, G.; Park, W.; Chilkoti, A.; et al. Overcoming Limitations in Nanoparticle Drug Delivery: Triggered, Intravascular Release to Improve Drug Penetration into Tumors. Cancer Res. 2012, 72, 5566-5575. 
33. Gillies, R. J.; Verduzco, D.; Gatenby, R. A. Evolutionary Dynamics of Carcinogenesis and why Targeted Therapy does not work. Nat. Rev. Cancer 2012, 12, 487-493. 
TOC Graphics

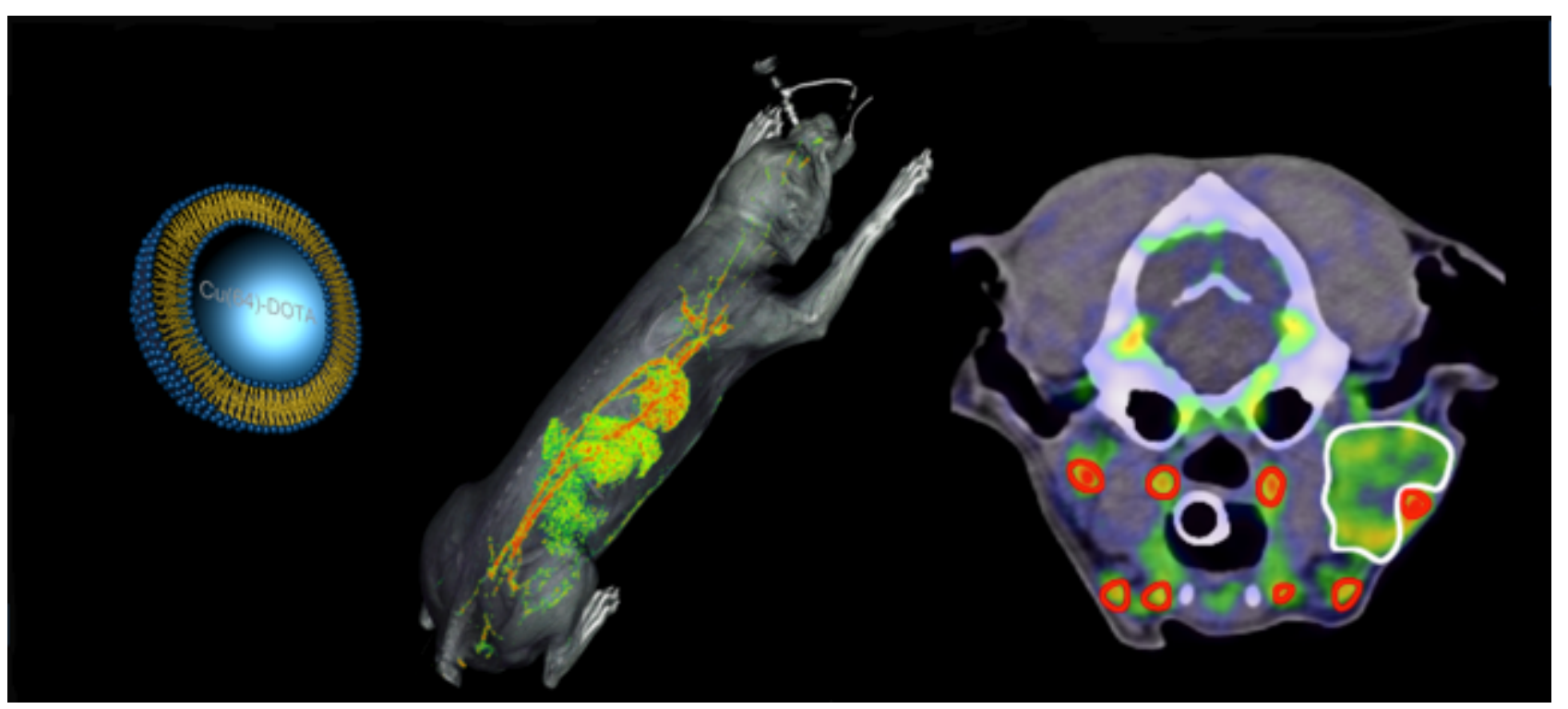




\section{Supporting information for:}

\section{Positron Emission Tomography based Elucidation of the Enhanced Permeability and Retention Effect in Dogs with Cancer using Copper-64 Liposomes}

Anders E.Hansen ${ }^{1,2}$, Anncatrine L. Petersen ${ }^{1}$, Jonas R. Henriksen ${ }^{1,3}$, Betina Boerresen, ${ }^{4}$ Palle Rasmussen ${ }^{1,5}$, Dennis R. Elema $^{1,5}$, Per Munch af Rosenschöld ${ }^{6}$, Annemarie T. Kristensen ${ }^{4}$, Andreas Kjarr ${ }^{2}$, Thomas L. Andresen ${ }^{1 *}$

${ }^{1}$ Center for Nanomedicine and Theranostics, DTU Nanotech, Technical University of Denmark, Building 423, DK-2800 Lyngby, Denmark; ${ }^{2}$ Department of Clinical Physiology, Nuclear Medicine \& PET, and Cluster for Molecular Imaging,

Rigshospitalet, Copenhagen University Hospital and Faculty of Health Sciences, University of Copenhagen,

Blegdamsvej 3B, DK-2200 Copenhagen, Denmark; ${ }^{3}$ DTU Chemistry, Technical University of Denmark, Building 206, DK-2800 Lyngby, Denmark; ${ }^{4}$ Department of Veterinary Clinical and Animal Sciences, Faculty of Health and Medical

Sciences, University of Copenhagen, Dyrlaegevej 16, DK-1870 Frederiksberg, Denmark; ${ }^{5}$ DTU Nutech, Hevesy Laboratory, Technical University of Denmark, Frederiksborgvej 399, DK-4000 Roskilde, Denmark; ${ }^{6}$ Radiation Medicine Research Center, Department of Radiation Oncology, Rigshospitalet, Copenhagen University Hospital, Blegdamsvej 9, DK-2100 Copenhagen, Denmark

E-mail: thomas.andresen@nanotech.dtu.dk

*Corresponding author contact details, Phone: +45 45258168, Fax: 4588 7762, E-mail:

Thomas.andresen@nanotech.dtu.dk 

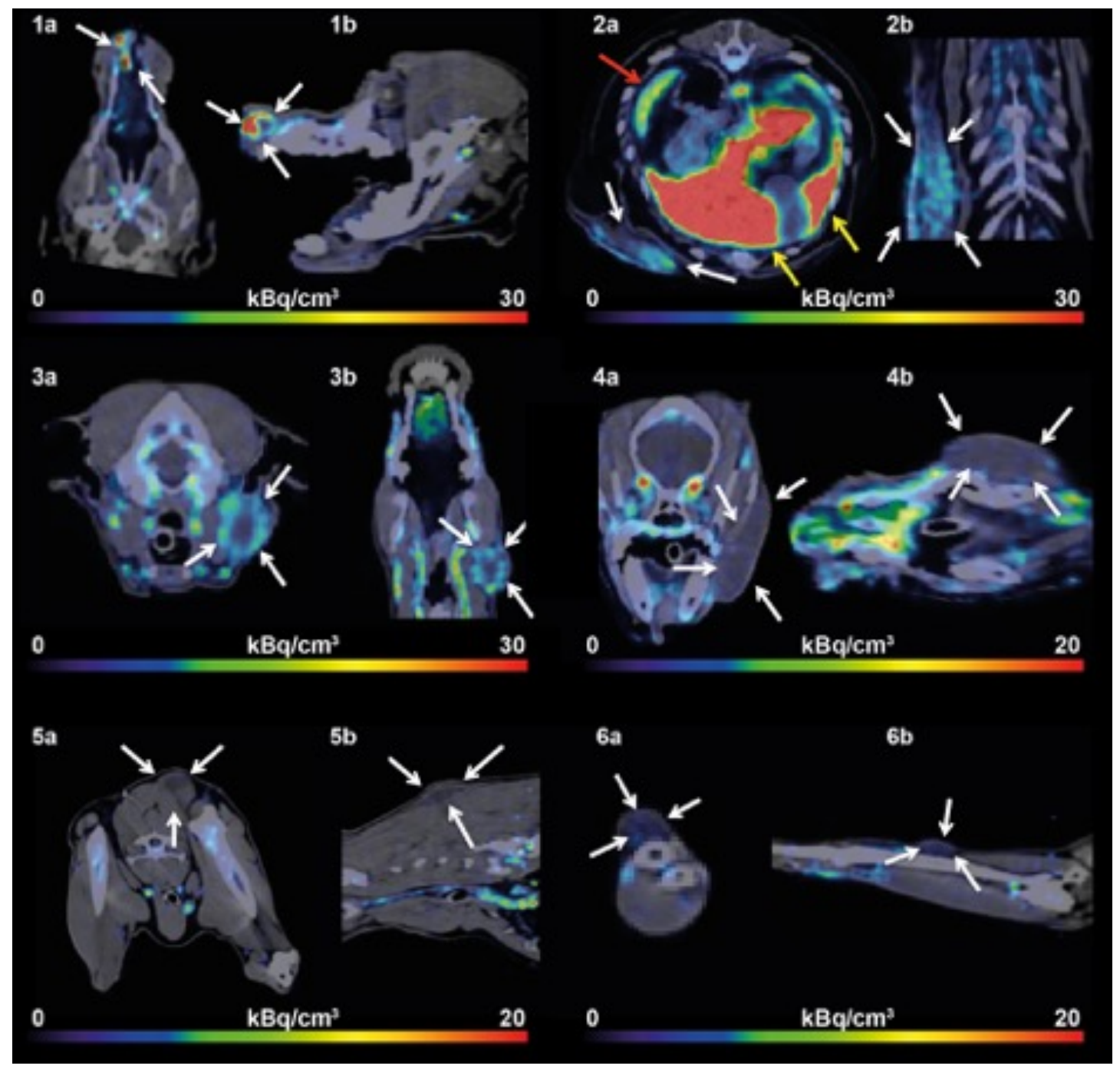

Figure S1. [Cu-64]-liposome PET/CT images (24 hours pi.) of dog 1 to 6 (numbering is identical to main text). Dog 1; intranasal squamous cell carcinoma, dog 2; mammary gland adenocarcinoma, dog 3 ; mandibular adenocarcinoma, $\operatorname{dog} 4$; masticatory muscle soft tissue sarcoma, dog 5; neck musculature soft tissue sarcoma, $\operatorname{dog} 6$; antebrachial liposarcoma. White arrows indicate tumors, red arrow indicate the spleen and yellow arrow the liver. The circulation time of [Cu-64]-liposomes in the blood can be appreciated by the high intravascular activity in larger blood vessels. For additional information on tumors, dogs and [Cu-64]liposome accumulation please refer to main text and Table 1 and 2. 


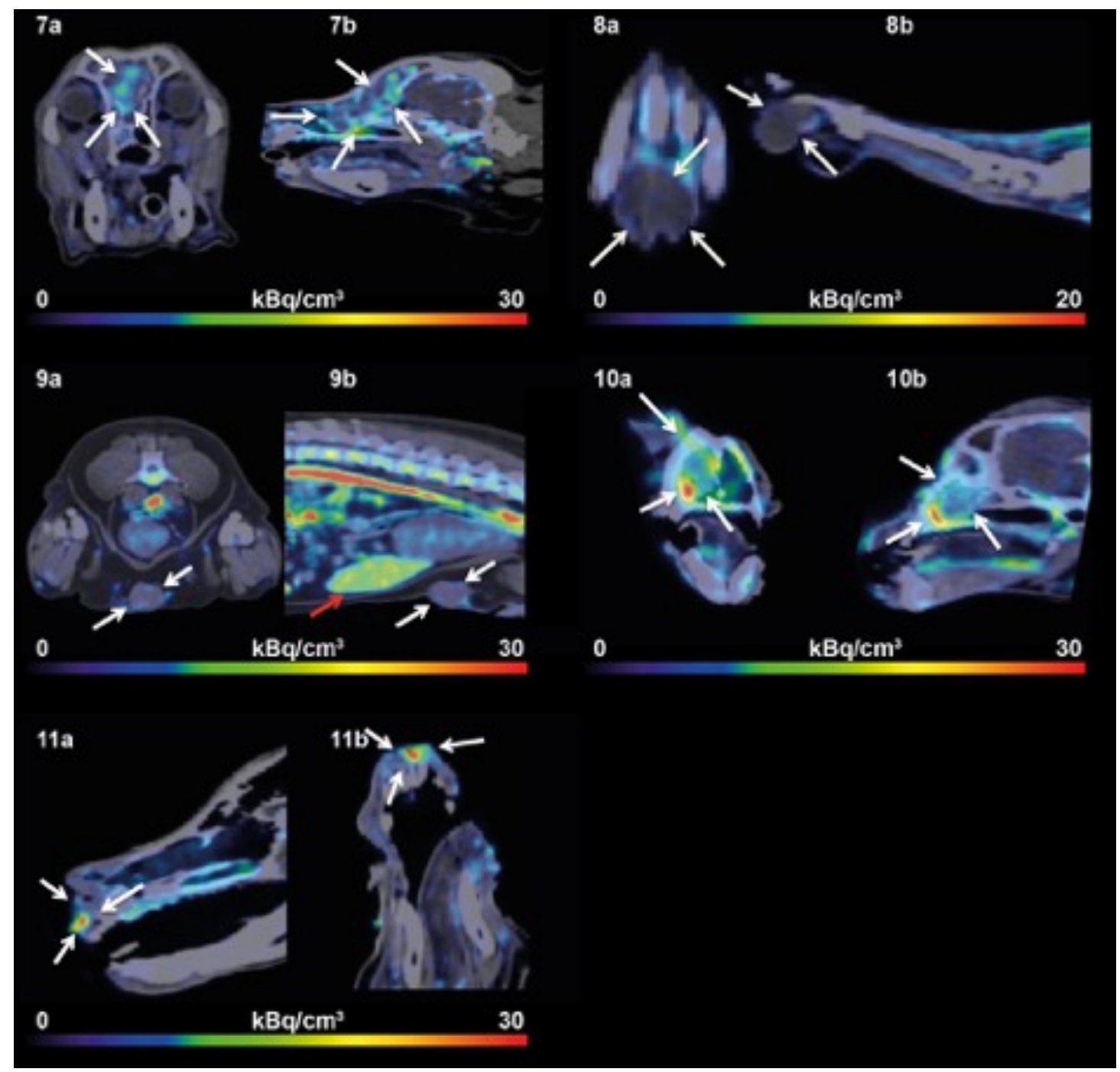

Figure S2. [Cu-64]-liposome PET/CT images (24 hours pi.) of dog 7 to 11 (numbering is identical to main text). Dog 7; intranasal transitional cell carcinoma, dog 8; digital soft tissue sarcoma in the front paw, dog 9; mammary gland adenocarcinoma, dog 10; intranasal squamous cell carcinoma, dog 11; intranasal adenocarcinoma. White arrows indicate tumors, red arrow indicates the spleen. The circulation time of [Cu64]-liposomes in the blood can be appreciated by the high intravascular activity in larger blood vessels. For information on tumors, dogs and [Cu-64]-liposome accumulation please refer to main text and Table 1 and 2. 

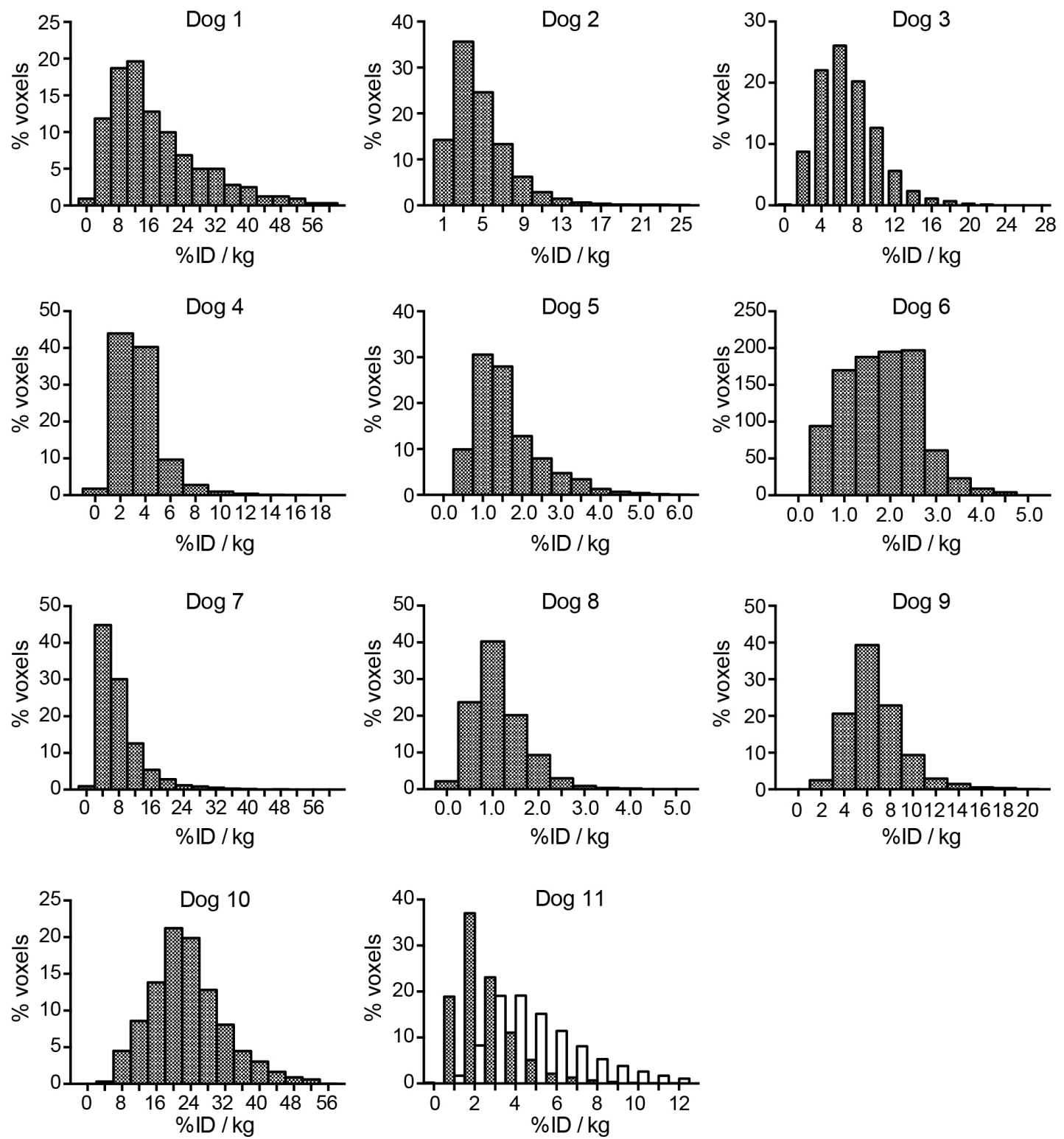

Figure S3. Histograms displaying voxel-wise uptake characteristics of $\% \mathrm{ID} / \mathrm{kg}$ [Cu-64]-liposome in tumor from included dogs. The white bars of dog 11 represents $\%$ ID $/ \mathrm{kg}$ corrected for $50 \%$ free-[Cu-64]-DOTA and gray bars uncorrected uptake of 50\% [Cu-64]-liposomes/50\% [Cu-64]-DOTA. 

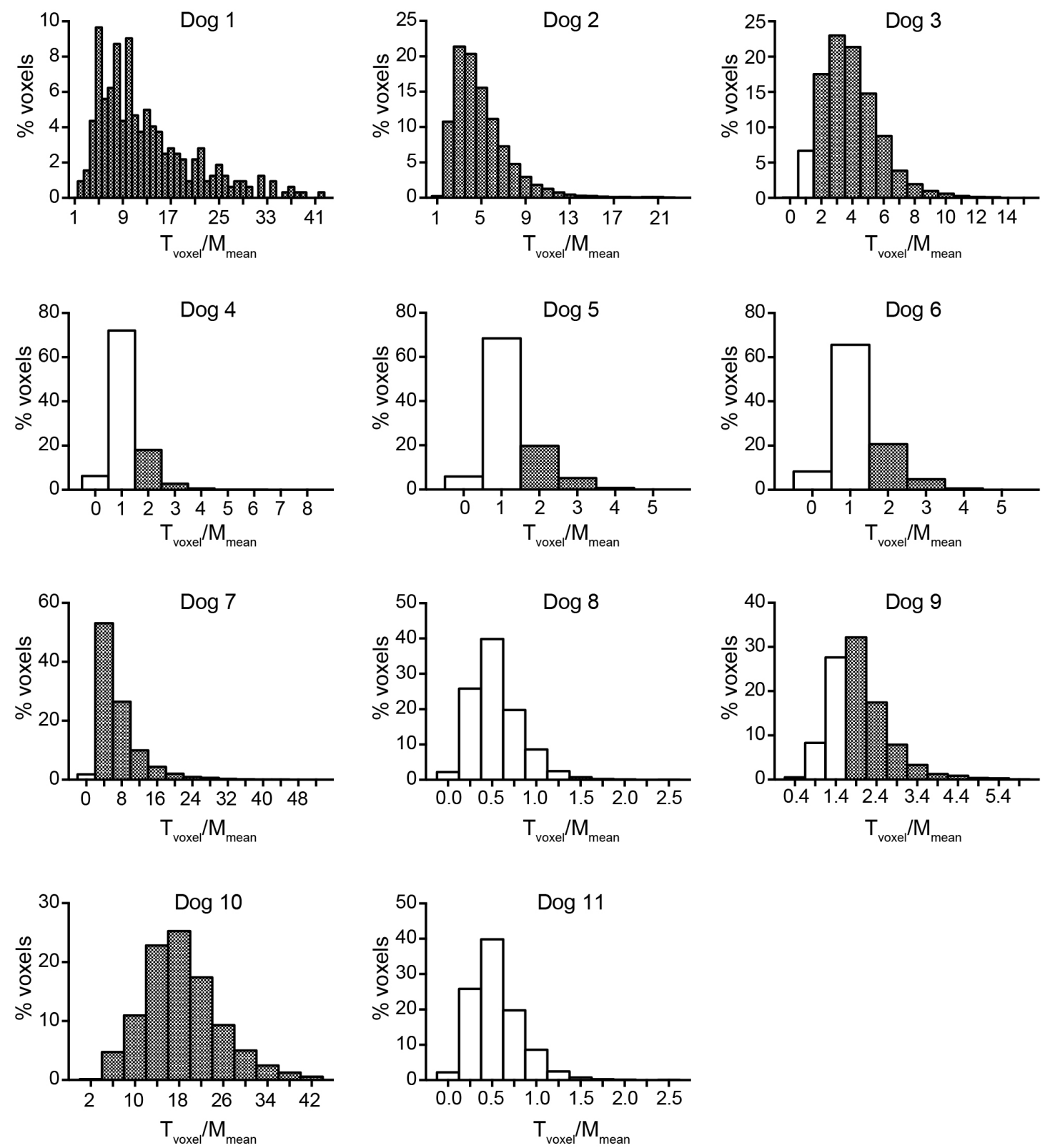

Figure S4. Histograms displaying voxel-wise tumor ${ }_{\text {voxel }}$-to-muscle $e_{\text {mean }}$ ratios [Cu-64]-liposome activity. The white bars represents voxels with a tumor-to-muscle ration below 1.5. For PET imaging a lesion-tobackground ratio above 1.5 is required for acceptable image contrast. 

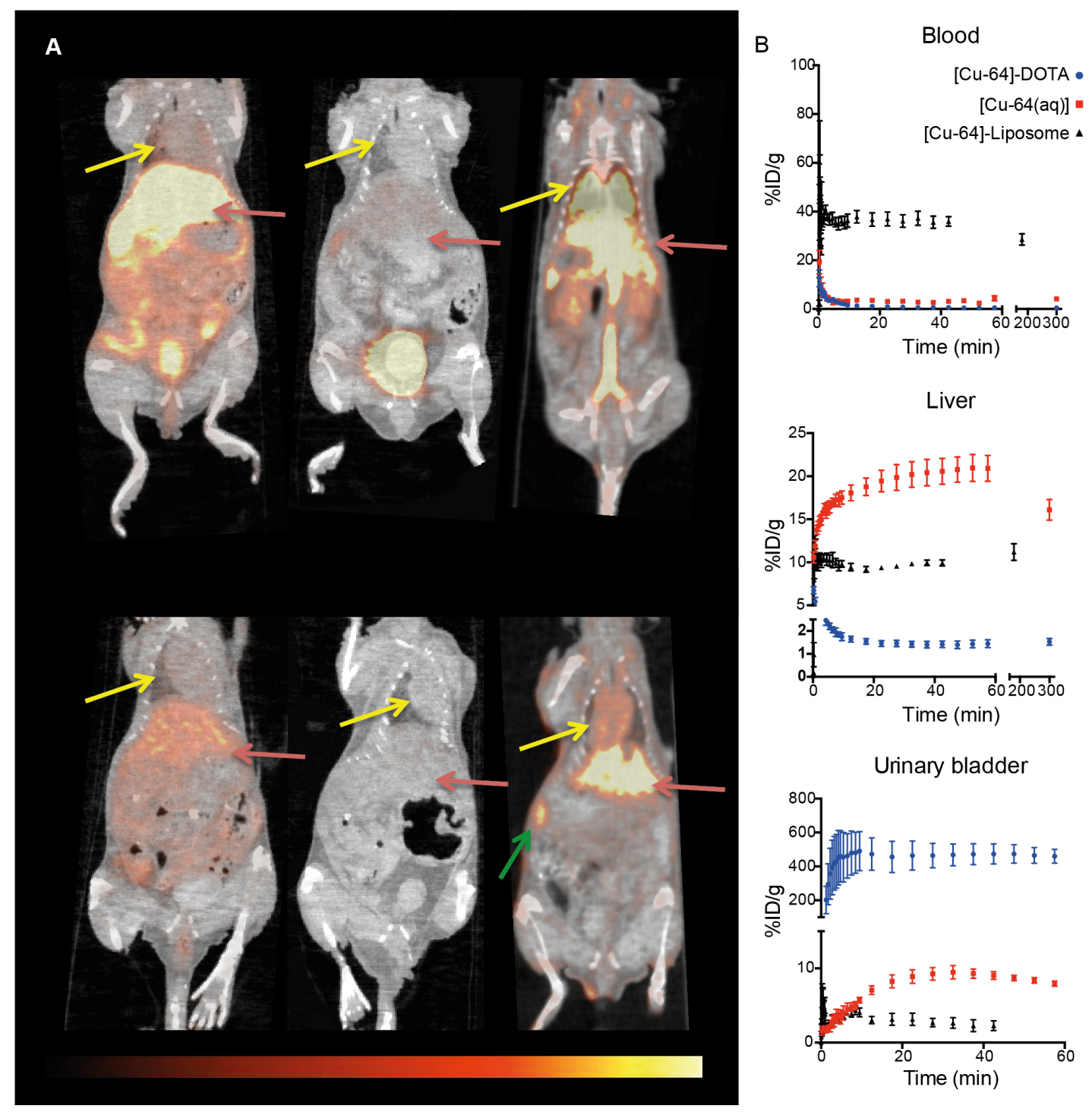

Figure S5. A: PET/CT images of [64-Cu(aq)] (left), [Cu-64]-DOTA (middle) and [Cu-64]-liposomes (right) in mice. Top row; images acquired 1 hour after intravenous administration of $10-12 \mathrm{MBq}(20 \mathrm{mg} \mathrm{lipid} / \mathrm{kg}$, encapsulation $>95 \%$ ). Bottom row; images acquired after a distribution period of 24 hours. B: Time activity curves (TAC) of dynamically acquired PET/CT displaying [Cu-64] activity in blood, liver and urinary bladder for [64-Cu(aq)] (red), [Cu-64]-DOTA (blue) and [64-Cu]-liposomes (black). Yellow arrows; heart, Red arrows; liver, Green arrows; spleen. The spleen could not be securely identified on the PET/CT scans of [Cu-64(aq)] and [Cu-64]-DOTA following poor image contrast. The blue arrow displays the high blood activity of [Cu-64]-liposomes in larger abdominal/pelvic vessels. The PET/CT images and TAC clearly displays the long circulating properties of [Cu-64]-liposomes compared to [Cu-64]-DOTA and [Cu-64(aq)]. The renal excretion of [Cu-64]-DOTA is extremely fast and very low urine activity levels of [Cu-64] are observed in mice injected with [Cu-64]-liposomes. Additionally, the mice injected with [Cu-64]-liposomes display a low and fast decreasing [Cu-64] activity in the bladder, which indicates that small amounts of unencapsulated [Cu-64]-DOTA is rapidly excreted and circulating liposomes are not leaking [Cu-64]-DOTA following the reduction in urine activity with continuing urine output (dilution). High hepatic uptake of free $[\mathrm{Cu}-64(\mathrm{aq})]$ is observed. This is expected following the high level of copper transporters in the liver. [Cu64]-liposomes displays an intermediate uptake compared to [Cu-64(aq)] and [Cu-64]-DOTA. The liver (red arrow) and spleen (green arrow) accumulation is compatible and indicate that [Cu-64]-liposomes are recognized by the retriculoendothelial systems (RES) and do not accumulate in the liver by a copper dependent transport mechanism. 


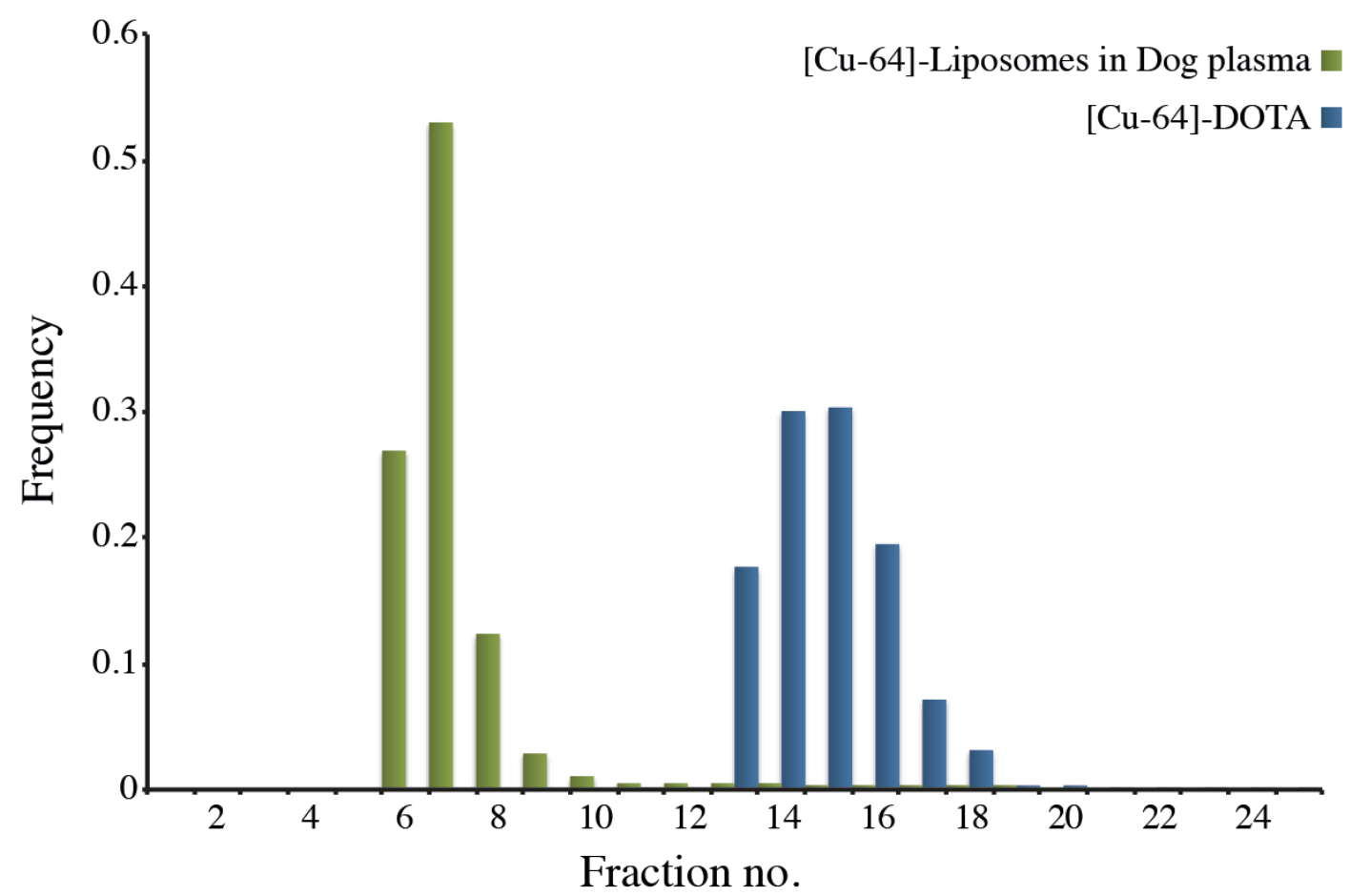

Figure S6. In vitro stability test of liposomes loaded with ${ }^{64} \mathrm{Cu}$ in dog plasma. Liposomes loaded with 500 $\mathrm{MBq}{ }^{64} \mathrm{Cu} / \mathrm{ml}$ was mixed with dog plasma in a $1: 1$ ratio, and incubated for $18 \mathrm{~h}$ at $37^{\circ} \mathrm{C}$. The liposomeplasma mixture $(200 \mu \mathrm{L})$ was separated on a size-exclusion column (Sephadex G50 fine, $25 \times 1.5 \mathrm{~cm}$ ) using a flow of $1 \mathrm{ml} / \mathrm{ml}$ (green fractions), and fractions were collected every $2^{\text {nd }} \mathrm{min}$. The ${ }^{64} \mathrm{Cu}$ activity of the different fractions was measured using a calibrated high-purity germanium detector (Princeton, Gammatech). Each sample was placed in a distance of $20 \mathrm{~cm}$ measured from the surface of the detector crystal. Energy and efficiency calibrations were performed prior to measuring using certified sealed radioactive sources of ${ }^{133} \mathrm{Ba}$ and ${ }^{152} \mathrm{Eu}$. The spectra were obtained and analyzed using Canberra Genie2K software. The ${ }^{64} \mathrm{Cu}$ activity of each sample was determined by integration of the $511 \mathrm{keV}$ annihilation peak and the activities were calculated based on the $20 \mathrm{~cm}$ efficiency calibration. [Cu-64]-DOTA was included as a control to determine retention-time of the free [Cu-64]-DOTA (blue fractions). The leakage of [Cu-64]-DOTA from the liposomes in dog plasma was $<3 \%$ (leakage $=\sum_{i=13}^{25} A_{\text {frac }}^{i} / \sum_{i=1}^{25} A_{\text {frac }}^{i}$, where $A_{\text {frac }}^{i}$ is the activity of the i'th fraction). The stability of liposomes loaded with ${ }^{64} \mathrm{Cu}$, was furthermore investigated in human and mice serum, and $<5 \%$ and $<2 \%$ leakage was found respectively. 